\title{
Monopeptide-based powder gelators for instant phase-selective gelation of aprotic aromatics and for toxic dye removal
}

Zhongyan Li, ${ }^{\dagger}$ Ziqing Luo, ${ }^{\dagger}$ Jialing Zhou, ${ }^{\dagger}$ Zecong Ye,${ }^{\ddagger}$ Guang-chuan Ou,${ }^{\dagger}$ Yanping Huo ${ }^{\ddagger}$ Lin Yuan* ${ }^{\dagger}$ and Huaqiang Zeng*\$

${ }^{\dagger}$ College of Chemistry and Bioengineering, Hunan University of Science and Engineering, Yongzhou, Hunan, China 425100

${ }^{*}$ Faculty of Chemical Engineering and Light Industry, Guangdong University of Technology, Guangzhou, Guangdong, China 510006

$\S$ Nanobio Lab, 31 Biopolis Way, The Nanos, Singapore 138669

Corresponding authors: hqzeng@ibn.a-star.edu.sg; tcyl431102@163.com

1. General Remarks

S2

2. Synthetic Scheme and Chemical Structures of PSOGs .53

3. Experimental Procedures and Compound Characterizations. .54

4. SEM images of the as-formed toluene and xylene gels by Fmoc-V-6.

5. ${ }^{1} \mathrm{H}$ NMR and ${ }^{13} \mathrm{C}$ NMR. S13 


\section{General Remarks}

All the reagents were obtained from commercial suppliers and used as received unless otherwise noted. The organic solvents were dried over anhydrous $\mathrm{Na}_{2} \mathrm{SO}_{4}$ for a minimum of 15 minutes before filtration. Flash column chromatography was performed using 200-300 mesh silica gel from QingDao Ocean Company. Chemical yield refers to pure isolated substances. ${ }^{1} \mathrm{H}$ and ${ }^{13} \mathrm{C}$ NMR spectra were recorded on a Bruker Avance HD400 spectrometer. The solvent signal of $\mathrm{CDCl}_{3}$ was referenced at $\delta$ $=7.26$ ppm. Coupling constants $(J$ values $)$ are reported in Hertz $(\mathrm{Hz}) .{ }^{1} \mathrm{H}$ NMR data are recorded in the order: chemical shift value, multiplicity (s, singlet; d, doublet; t, triplet; q, quartet; m, multiplet; br, broad), number of protons that gave rise to the signal and coupling constant, where applicable. ${ }^{13} \mathrm{C}$ spectra are proton-decoupled and recorded on Bruker Avance $\mathrm{HD} 400(100 \mathrm{MHz})$. The solvent, $\mathrm{CDCl}_{3}$, was referenced at $\delta=77 \mathrm{ppm}$. $\mathrm{CDCl}_{3}$ (99.8\%-Deuterated) was purchased from Alfa and used without further purification. Mass spectra were acquired with an SHIMADZU LCMS-8030. 
Scheme S1: Synthetic route that generates phase-selective organogelator library
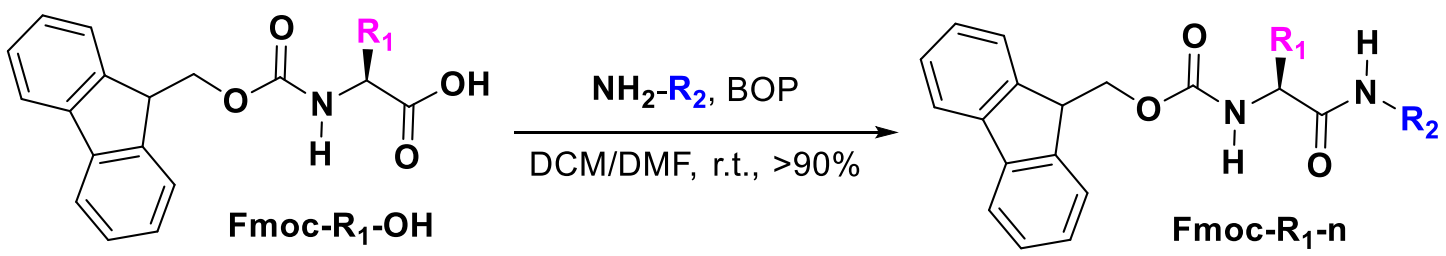

$\mathrm{n}=4,6,8,10,12,14$ and 16

$\mathrm{R}_{1}=\mathrm{CH}_{3}(\mathbf{A}) ; \mathrm{CH}\left(\mathrm{CH}_{3}\right)_{2}(\mathbf{V}) ; \mathrm{CH}_{2} \mathrm{CH}\left(\mathrm{CH}_{3}\right)_{2}(\mathbf{L}) ; \mathrm{CH}\left(\mathrm{CH}_{3}\right) \mathrm{C}_{2} \mathrm{H}_{5}(\mathbf{I}) ; \mathrm{CH}_{2} \mathrm{C}_{6} \mathrm{H}_{5}(\mathbf{F})$

$\mathbf{R}_{\mathbf{2}}=n-\mathrm{C}_{4} \mathrm{H}_{9} ; n-\mathrm{C}_{6} \mathrm{H}_{13} ; n-\mathrm{C}_{8} \mathrm{H}_{17} ; n-\mathrm{C}_{10} \mathrm{H}_{21}, n-\mathrm{C}_{12} \mathrm{H}_{25} ; n-\mathrm{C}_{14} \mathrm{H}_{29}, n-\mathrm{C}_{16} \mathrm{H}_{33}$<smiles>[R]NC(=O)[C@H](C)NC(=O)OCC1c2ccccc2-c2ccccc21</smiles>

Fmoc-A-4

Fmoc-A-6

Fmoc-A-8

Fmoc-A-10

Fmoc-A-12

Fmoc-A-14

Fmoc-A-16

$\mathrm{R}=n-\mathrm{C}_{4} \mathrm{H}_{9}$

$\mathrm{R}=n-\mathrm{C}_{6} \mathrm{H}_{13}$

$\mathrm{R}=n-\mathrm{C}_{8} \mathrm{H}_{17}$

$\mathrm{R}=n-\mathrm{C}_{10} \mathrm{H}_{21}$

$\mathrm{R}=n-\mathrm{C}_{12} \mathrm{H}_{25}$

$\mathrm{R}=n-\mathrm{C}_{14} \mathrm{H}_{29}$

$\mathrm{R}=n-\mathrm{C}_{16} \mathrm{H}_{33}$<smiles>[R]NC(=O)[C@H](NC(=O)OCC1c2ccccc2-c2ccccc21)C(C)C</smiles>

\section{Fmoc-V-4}

Fmoc-V-6

Fmoc-V-8

Fmoc-V-10

Fmoc-V-12

Fmoc-V-14

$\mathrm{R}=n-\mathrm{C}_{4} \mathrm{H}_{9}$

$\mathrm{R}=n-\mathrm{C}_{6} \mathrm{H}_{13}$

$\mathrm{R}=n-\mathrm{C}_{8} \mathrm{H}_{17}$

$\mathrm{R}=n-\mathrm{C}_{10} \mathrm{H}_{21}$

$\mathrm{R}=n-\mathrm{C}_{12} \mathrm{H}_{25}$

Fmoc-V-16

$\mathrm{R}=n-\mathrm{C}_{14} \mathrm{H}_{29}$<smiles>[R]NC(=O)[C@H](CC(C)C)NC(=O)OCC1c2ccccc2-c2ccccc21</smiles>

Fmoc-L-4

Fmoc-L-6

$\mathrm{R}=n-\mathrm{C}_{16} \mathrm{H}_{33}$

$\quad \mathrm{R}=n-\mathrm{C}_{6} \mathrm{H}_{13}$

Fmoc-L-8 $\quad \mathrm{R}=n-\mathrm{C}_{8} \mathrm{H}_{17}$

Fmoc-L-10 $\mathrm{R}=n-\mathrm{C}_{10} \mathrm{H}_{21}$

Fmoc-L-12 $\mathrm{R}=n-\mathrm{C}_{12} \mathrm{H}_{25}$

Fmoc-L-14 $\mathrm{R}=n-\mathrm{C}_{14} \mathrm{H}_{29}$

Fmoc-L-16 $\mathrm{R}=n-\mathrm{C}_{16} \mathrm{H}_{33}$<smiles>[R]NC(=O)[C@H](NC(=O)OCC1c2ccccc2-c2ccccc21)C(C)CC</smiles>

Fmoc-I-4

Fmoc-I-6

$\mathrm{R}=n-\mathrm{C}_{4} \mathrm{H}_{9}$

$\mathrm{R}=n-\mathrm{C}_{6} \mathrm{H}_{13}$

Fmoc-I-8 $\quad \mathrm{R}=n-\mathrm{C}_{8} \mathrm{H}_{17}$

Fmoc-l-10 $\quad \mathrm{R}=n-\mathrm{C}_{10} \mathrm{H}_{21}$

Fmoc-l-12 $\mathrm{R}=n-\mathrm{C}_{12} \mathrm{H}_{25}$

Fmoc-l-14 $\mathrm{R}=n-\mathrm{C}_{14} \mathrm{H}_{29}$

Fmoc-I-16 $\mathrm{R}=n-\mathrm{C}_{16} \mathrm{H}_{33}$<smiles>[R]NC(=O)[C@H](Cc1ccccc1)NC(=O)OCC1c2ccccc2-c2ccccc21</smiles>

Fmoc-F-4 Fmoc-F-6

$\mathrm{R}=n-\mathrm{C}_{4} \mathrm{H}_{9}$

$\mathrm{R}=n-\mathrm{C}_{6} \mathrm{H}_{13}$

Fmoc-F-8 $\quad \mathrm{R}=n-\mathrm{C}_{8} \mathrm{H}_{17}$

Fmoc-F-10 $\mathrm{R}=n-\mathrm{C}_{10} \mathrm{H}_{21}$

Fmoc-F-12 $\mathrm{R}=n-\mathrm{C}_{12} \mathrm{H}_{25}$

Fmoc-F-14 $\mathrm{R}=n-\mathrm{C}_{14} \mathrm{H}_{29}$

Fmoc-F-16 $\mathrm{R}=n-\mathrm{C}_{16} \mathrm{H}_{33}$ 


\section{Experimental Procedures and Compound Characterizations}

For syntheses of Fmoc-A-n, Fmoc-V-n, Fmoc-A-n, Fmoc-L-n, Fmoc-I-n and Fmoc-F-n (n = 4, 6, 8, 10), see Ren, C. L.; Ng, G. H. B.; Wu, H.; Chan, K.-H.; Shen, J.; Teh, C.; Ying, J. Y.; Zeng, H. Q. Chem. Mater. 2016, 28, 4001-4008

\section{Fmoc-A-12}

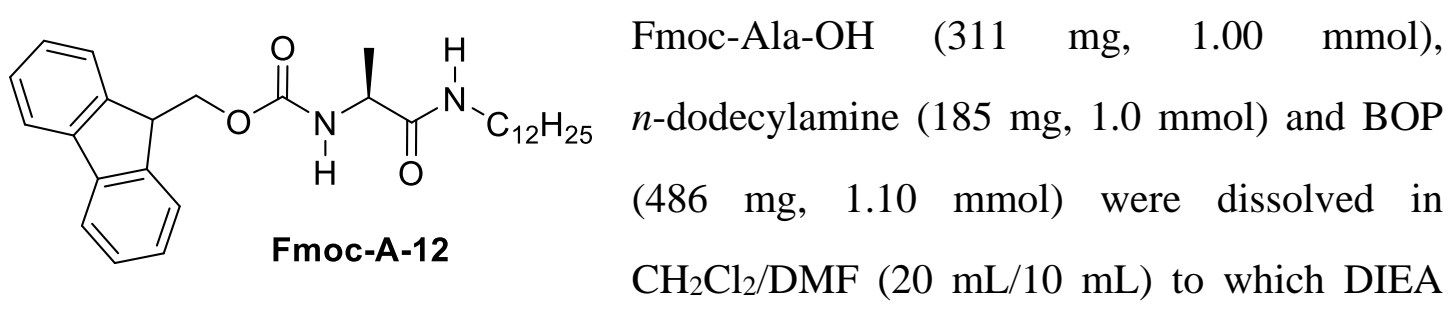

$(0.39 \mathrm{ml}, 2.20 \mathrm{mmol})$ was added. The reaction mixture was stirred for $20 \mathrm{~h}$ at room temperature. Solvent was removed in vacuo and the crude product was dissolved in $\mathrm{CH}_{2} \mathrm{Cl}_{2}(50 \mathrm{~mL})$, washed with water $(2 \times 50 \mathrm{~mL})$ and dried over $\mathrm{Na}_{2} \mathrm{SO}_{4}$ to give the crude product, which was subject to flash column chromatography $\left(\mathrm{MeOH}: \mathrm{CH}_{2} \mathrm{Cl}_{2}=\right.$ 1:25, v:v) to yield pure product F-A-12 as a white solid. Yield: $445 \mathrm{mg}, 93 \% .{ }^{1} \mathrm{H}$ NMR (400 MHz, $\left.\mathrm{CDCl}_{3}\right) \delta 7.75(\mathrm{~d}, J=7.5 \mathrm{~Hz}, 2 \mathrm{H}), 7.57(\mathrm{~d}, J=7.2 \mathrm{~Hz}, 2 \mathrm{H}), 7.39$ (t, $J=7.3 \mathrm{~Hz}, 2 \mathrm{H}), 7.29(\mathrm{t}, J=7.3 \mathrm{~Hz}, 2 \mathrm{H}), 6.35(\mathrm{~s}, 1 \mathrm{H}), 5.67(\mathrm{~d}, J=6.1 \mathrm{~Hz}, 1 \mathrm{H}), 4.37$ $(\mathrm{d}, J=6.3 \mathrm{~Hz}, 2 \mathrm{H}), 4.29-4.13(\mathrm{~m}, 2 \mathrm{H}), 3.28-3.14(\mathrm{~m}, 2 \mathrm{H}), 1.50-1.42(\mathrm{~m}, 2 \mathrm{H}), 1.38$ (d, $J=5.0 \mathrm{~Hz}, 3 \mathrm{H}), 1.32-1.18(\mathrm{~m}, 18 \mathrm{H}), 0.92-0.85(\mathrm{~m}, 3 \mathrm{H}) .{ }^{13} \mathrm{C} \mathrm{NMR}\left(100 \mathrm{MHz}, \mathrm{CDCl}_{3}\right)$ $\delta 172.28,156.12,143.76,141.32,127.79,127.12,125.08,120.03,67.08,50.57,47.12$, $39.67,31.94,29.67,29.62,29.57,29.48,29.38,29.30,26.89,22.72,18.89$, 14.16 . MS-ESI: calculated for $[\mathrm{M}+\mathrm{Na}]^{+}\left(\mathrm{C}_{30} \mathrm{H}_{42} \mathrm{O}_{3} \mathrm{~N}_{2} \mathrm{Na}\right): \mathrm{m} / \mathrm{z}$ 501.31, found: m/z 501.26. 
The following molecules were prepared using the same synthetic procedure as F-A-12.

\section{Fmoc-A-14}<smiles>CCCCCCCCNC(=O)[C@H](C)NC(=O)OCC1c2ccccc2-c2ccccc21</smiles>

Yield: $480 \mathrm{mg}$, 95\%. ${ }^{1} \mathrm{H}$ NMR (400 MHz, $\left.\mathrm{CDCl}_{3}\right) \delta 7.77(\mathrm{~d}, J=7.5 \mathrm{~Hz}, 2 \mathrm{H}), 7.58(\mathrm{~d}, J=$ $7.4 \mathrm{~Hz}, 2 \mathrm{H}), 7.40$ (t, $J=7.4 \mathrm{~Hz}, 2 \mathrm{H}), 7.31(\mathrm{t}, J=$ $7.4 \mathrm{~Hz}, 2 \mathrm{H}), 6.01(\mathrm{~s}, 1 \mathrm{H}), 5.40(\mathrm{~d}, J=6.2 \mathrm{~Hz}$, $1 \mathrm{H}), 4.40(\mathrm{~d}, J=6.4 \mathrm{~Hz}, 2 \mathrm{H}), 4.21(\mathrm{t}, J=6.9 \mathrm{~Hz}, 2 \mathrm{H}), 3.24(\mathrm{q}, J=6.4 \mathrm{~Hz}, 2 \mathrm{H})$, 1.53-1.44 (m, 2H), 1.39 (d, J = 6.6 Hz, 3H), 1.33-1.20 (m, 22H), 0.91-0.85 (m, 3H). ${ }^{13} \mathrm{C}$ NMR $\left(100 \mathrm{MHz}, \mathrm{CDCl}_{3}\right) \delta 172.13,156.08,143.76,141.33,127.79,127.11$, $125.07,120.04,67.08,50.58,47.13,39.66,31.95,29.72,29.71,29.68,29.62,29.58$, $29.51,29.39,29.30,26.89,22.73,18.89,14.16$. MS-ESI: calculated for $[\mathrm{M}+\mathrm{Na}]^{+}$ $\left(\mathrm{C}_{32} \mathrm{H}_{46} \mathrm{O}_{3} \mathrm{~N}_{2} \mathrm{Na}\right): \mathrm{m} / \mathrm{z}$ 529.34, found: $\mathrm{m} / \mathrm{z} 529.25$.

\section{Fmoc-A-16}

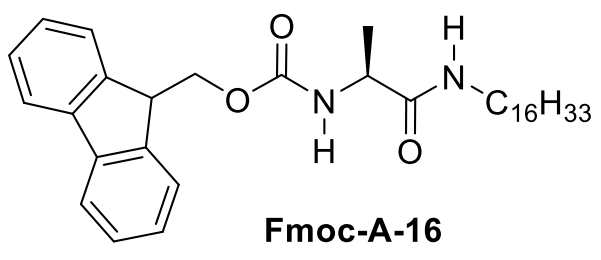

Yield: $513 \mathrm{mg}, 96 \% .{ }^{1} \mathrm{H}$ NMR (400 MHz, $\left.\mathrm{CDCl}_{3}\right) \delta 7.76(\mathrm{~d}, J=7.5 \mathrm{~Hz}, 2 \mathrm{H}), 7.58(\mathrm{~d}, J=$ $7.4 \mathrm{~Hz}, 2 \mathrm{H}), 7.40(\mathrm{t}, J=7.4 \mathrm{~Hz}, 2 \mathrm{H}), 7.31(\mathrm{t}, J=$ $7.4 \mathrm{~Hz}, 2 \mathrm{H}), 6.05$ (s, 1H), 5.44 (d, $J=6.2 \mathrm{~Hz}$, $1 \mathrm{H}), 4.39(\mathrm{~d}, J=6.6 \mathrm{~Hz}, 2 \mathrm{H}), 4.20(\mathrm{t}, J=6.9 \mathrm{~Hz}, 2 \mathrm{H}), 3.24(\mathrm{q}, J=6.4 \mathrm{~Hz}, 2 \mathrm{H})$, $1.52-1.43(\mathrm{~m}, 2 \mathrm{H}), 1.38(\mathrm{~d}, J=6.6 \mathrm{~Hz}, 3 \mathrm{H}), 1.30-1.19(\mathrm{~m}, 26 \mathrm{H}), 0.91-0.85(\mathrm{~m}, 3 \mathrm{H})$. ${ }^{13} \mathrm{C}$ NMR $\left(100 \mathrm{MHz}, \mathrm{CDCl}_{3}\right) \delta 172.04,156.05,143.77,141.34,127.79,127.12$, $125.06,120.04,67.06,50.60,47.15,39.66,31.95,29.72,29.69,29.67,29.62,29.57$, $29.51,29.39,29.29,26.88,22.72,18.80,14.16$. MS-ESI: calculated for $[\mathrm{M}+\mathrm{Na}]^{+}$ $\left(\mathrm{C}_{34} \mathrm{H}_{50} \mathrm{O}_{3} \mathrm{~N}_{2} \mathrm{Na}\right): \mathrm{m} / \mathrm{z}$ 557.37, found: $\mathrm{m} / \mathrm{z}$ 505.26. 


\section{Fmoc-V-12}

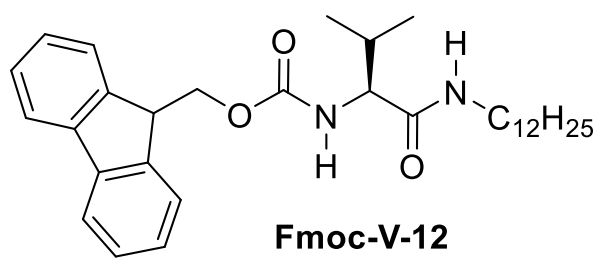

Yield: $476 \mathrm{mg}, 94 \% .{ }^{1} \mathrm{H}$ NMR (400 MHz, $\left.\mathrm{CDCl}_{3}\right) \delta 7.76(\mathrm{~d}, J=7.5 \mathrm{~Hz}, 2 \mathrm{H}), 7.58(\mathrm{~d}, J=$ $7.5 \mathrm{~Hz}, 2 \mathrm{H}), 7.40(\mathrm{t}, J=7.5 \mathrm{~Hz}, 2 \mathrm{H}), 7.31(\mathrm{t}, J=$ $7.4 \mathrm{~Hz}, 2 \mathrm{H}), 5.95(\mathrm{~s}, 1 \mathrm{H}), 5.51(\mathrm{~d}, J=8.9 \mathrm{~Hz}$, $1 \mathrm{H}), 4.39(\mathrm{dt}, J=24.4,10.0 \mathrm{~Hz}, 2 \mathrm{H}), 4.21(\mathrm{t}, J=7.0 \mathrm{~Hz}, 1 \mathrm{H}), 3.90(\mathrm{t}, J=7.8 \mathrm{~Hz}, 1 \mathrm{H})$, 3.38-3.09 (m, 2H), 2.18-2.02 (m, 1H), 1.56-1.39 (m, 2H), 1.34-1.17 (m, 18H), 0.99-0.84 (m, 9H). ${ }^{13} \mathrm{C}$ NMR $\left(100 \mathrm{MHz}, \mathrm{CDCl}_{3}\right) \delta 171.16,156.54,143.84,143.79$, $141.31,127.76,127.12,125.09,120.01,67.07,60.71,54.66,47.17,42.83,39.60$, $36.81,31.94,31.15,29.66,29.61,29.56,29.53,29.37,29.27,26.92,22.71,19.26$, 18.11, 14.15. MS-ESI: calculated for $[\mathrm{M}+\mathrm{Na}]^{+}\left(\mathrm{C}_{32} \mathrm{H}_{46} \mathrm{O}_{3} \mathrm{~N}_{2} \mathrm{Na}\right): \mathrm{m} / \mathrm{z} 529.34$, found: $\mathrm{m} / \mathrm{z} 529.23$.

\section{Fmoc-V-14}

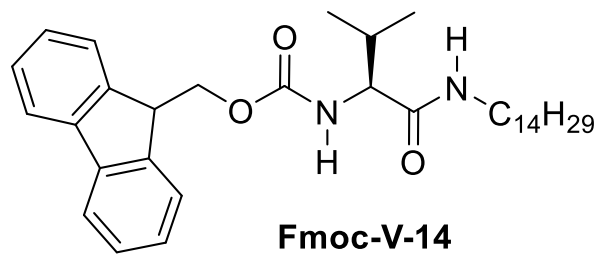

Yield: $513 \mathrm{mg}, 96 \% .{ }^{1} \mathrm{H}$ NMR $(400 \mathrm{MHz}$, $\left.\mathrm{CDCl}_{3}\right) \delta 7.76(\mathrm{~d}, J=7.5 \mathrm{~Hz}, 2 \mathrm{H}), 7.58(\mathrm{~d}, J=$ $7.4 \mathrm{~Hz}, 2 \mathrm{H}), 7.40(\mathrm{t}, J=7.4 \mathrm{~Hz}, 2 \mathrm{H}), 7.31(\mathrm{t}, J=$ $7.4 \mathrm{~Hz}, 2 \mathrm{H}), 5.93(\mathrm{~s}, 1 \mathrm{H}), 5.47(\mathrm{~d}, J=8.7 \mathrm{~Hz}$, $1 \mathrm{H}), 4.48-4.31(\mathrm{~m}, 2 \mathrm{H}), 4.21(\mathrm{t}, J=6.9 \mathrm{~Hz}, 1 \mathrm{H}), 3.90(\mathrm{t}, J=7.8 \mathrm{~Hz}, 1 \mathrm{H}), 3.36-3.12$ $(\mathrm{m}, 2 \mathrm{H}), 2.20-2.04(\mathrm{~m}, 1 \mathrm{H}), 1.55-1.41(\mathrm{~m}, 2 \mathrm{H}), 1.33-1.20(\mathrm{~m}, 22 \mathrm{H}), 0.99-0.84(\mathrm{~m}$, 9H). ${ }^{13} \mathrm{C} \mathrm{NMR}\left(100 \mathrm{MHz}, \mathrm{CDCl}_{3}\right) \delta 171.08,156.48,143.83,143.80,141.33,127.76$, $127.12,125.09,120.02,67.05,60.73,47.19,39.61,31.95,31.10,29.72,29.71,29.68$, $29.61,29.56,29.54,29.40,29.27,26.91,22.73,19.26,18.08,14.17$. MS-ESI: calculated for $[\mathrm{M}+\mathrm{Na}]^{+}\left(\mathrm{C}_{34} \mathrm{H}_{50} \mathrm{O}_{3} \mathrm{~N}_{2} \mathrm{Na}\right): \mathrm{m} / \mathrm{z}$ 557.37, found: $\mathrm{m} / \mathrm{z}$ 557.26. 


\section{Fmoc-V-16}

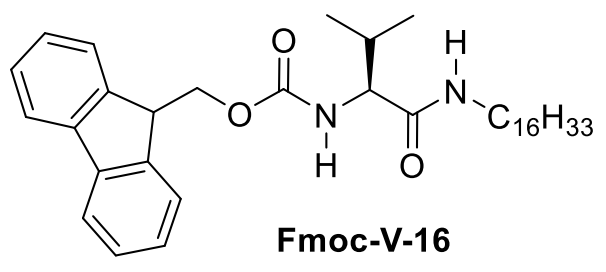

Yield: $545 \mathrm{mg}$, 97\%. ${ }^{1} \mathrm{H}$ NMR (400 MHz, $\left.\mathrm{CDCl}_{3}\right) \delta 7.76(\mathrm{~d}, J=7.5 \mathrm{~Hz}, 2 \mathrm{H}), 7.58(\mathrm{~d}, J=$ $7.3 \mathrm{~Hz}, 2 \mathrm{H}), 7.39(\mathrm{t}, J=7.4 \mathrm{~Hz}, 2 \mathrm{H}), 7.30(\mathrm{t}, J=$ $7.3 \mathrm{~Hz}, 2 \mathrm{H}), 6.00(\mathrm{~s}, 1 \mathrm{H}), 5.51(\mathrm{~d}, J=8.7 \mathrm{~Hz}$, 1H), 4.46-4.30 (m, 2H), $4.20(\mathrm{t}, J=6.9 \mathrm{~Hz}, 1 \mathrm{H}), 3.90(\mathrm{t}, J=7.8 \mathrm{~Hz}, 1 \mathrm{H}), 3.34-3.11$ $(\mathrm{m}, 2 \mathrm{H}), 2.15-2.05(\mathrm{~m}, 1 \mathrm{H}), 1.51-1.42(\mathrm{~m}, 2 \mathrm{H}), 1.35-1.18(\mathrm{~m}, 26 \mathrm{H}), 0.99-0.83(\mathrm{~m}$, 9H). ${ }^{13} \mathrm{C}$ NMR $\left(100 \mathrm{MHz}, \mathrm{CDCl}_{3}\right) \delta 171.10,156.51,143.84,143.80,141.33,127.76$, 127.12, 125.09, 120.01, 67.06, 60.74, 47.19, 39.60, 31.95, 31.11, 29.72, 29.69, 29.67, 29.61, 29.56, 29.54, 29.39, 29.27, 26.91, 22.72, 19.25, 18.10, 14.16. MS-ESI: calculated for $[\mathrm{M}+\mathrm{Na}]^{+}\left(\mathrm{C}_{36} \mathrm{H}_{54} \mathrm{O}_{3} \mathrm{~N}_{2} \mathrm{Na}\right): \mathrm{m} / \mathrm{z}$ 585.40, found: $\mathrm{m} / \mathrm{z}$ 585.30.

\section{Fmoc-L-12}

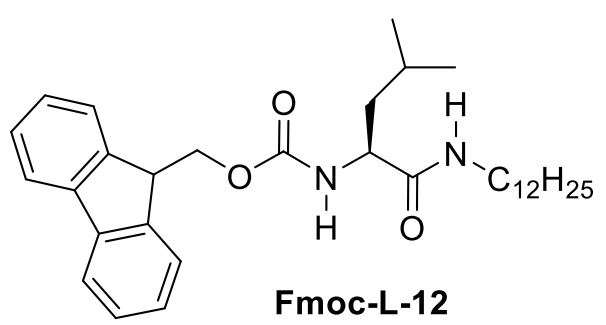

Yield: $478 \mathrm{mg}$, 92\%. ${ }^{1} \mathrm{H}$ NMR (400 MHz, $\left.\mathrm{CDCl}_{3}\right) \delta 7.75(\mathrm{~d}, J=7.5 \mathrm{~Hz}, 2 \mathrm{H}), 7.56(\mathrm{~d}, J=$ $7.4 \mathrm{~Hz}, 2 \mathrm{H}), 7.39$ (t, $J=7.4 \mathrm{~Hz}, 2 \mathrm{H}), 7.30(\mathrm{t}, J=$ $7.4 \mathrm{~Hz}, 2 \mathrm{H}), 6.02(\mathrm{~s}, 1 \mathrm{H}), 5.27(\mathrm{~d}, J=8.1 \mathrm{~Hz}$, $1 \mathrm{H}), 4.44-4.35(\mathrm{~m}, 2 \mathrm{H}), 4.24-4.08(\mathrm{~m}, 2 \mathrm{H})$, 3.30-3.16 (m, 2H), 1.72-1.60 (m, 2H), 1.58-1.50 (m, 1H), 1.49-1.40 (m, 2H), 1.30-1.21 (m, 18H), 0.99-0.84 (m, 9H). $\left.{ }^{13} \mathrm{C} \mathrm{NMR} \mathrm{(100} \mathrm{MHz,} \mathrm{CDCl}_{3}\right) \delta$ 171.93, 156.23, 143.72, 143.68, 141.28, 127.71, 127.06, 125.00, 119.98, 66.94, 53.54, 47.12, $41.46,39.58,31.89,29.66,29.65,29.62,29.56,29.51,29.43,29.33,29.23,26.82$, 24.66, 22.87, 22.67, 22.06, 14.11. MS-ESI: calculated for $[\mathrm{M}+\mathrm{Na}]^{+}\left(\mathrm{C}_{33} \mathrm{H}_{48} \mathrm{O}_{3} \mathrm{~N}_{2} \mathrm{Na}\right)$ : $\mathrm{m} / \mathrm{z}$ 543.36, found: $\mathrm{m} / \mathrm{z}$ 543.28. 


\section{Fmoc-L-14}

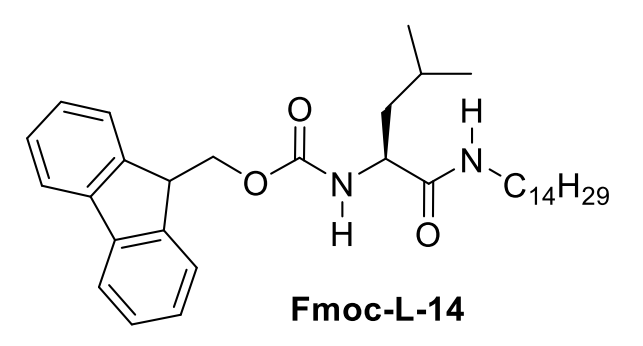

Yield: $504 \mathrm{mg}$, 92\%. ${ }^{1} \mathrm{H}$ NMR (400 MHz, $\left.\mathrm{CDCl}_{3}\right) \delta 7.76(\mathrm{~d}, J=7.5 \mathrm{~Hz}, 2 \mathrm{H}), 7.57(\mathrm{~d}, J=$ $7.4 \mathrm{~Hz}, 2 \mathrm{H}), 7.40(\mathrm{t}, J=7.4 \mathrm{~Hz}, 2 \mathrm{H}), 7.31(\mathrm{t}, J=$ $7.4 \mathrm{~Hz}, 2 \mathrm{H}), 6.03(\mathrm{~s}, 1 \mathrm{H}), 5.28(\mathrm{~d}, J=8.1 \mathrm{~Hz}$, $1 \mathrm{H}), 4.46-4.35(\mathrm{~m}, 2 \mathrm{H}), 4.25-4.08(\mathrm{~m}, 2 \mathrm{H})$, 3.30-3.15 (m, 2H), 1.72-1.60 (m, 2H), 1.58-1.51 (m, 1H), 1.50-1.41 (m, 2H), 1.32-1.21 (m, 22H), 0.99-0.84 (m, 9H). $\left.{ }^{13} \mathrm{C} \mathrm{NMR} \mathrm{(100} \mathrm{MHz,} \mathrm{CDCl}_{3}\right) \delta$ 171.96, $156.27,143.75,143.72,141.31,127.74,127.09,125.03,125.09,120.01,66.97,53.57$, $47.15,41.49,39.61,31.93,29.69,29.68,29.65,29.59,29.55,29.47,29.37,29.26$, $26.85,24.69,22.90,22.70,22.09$, 14.14. MS-ESI: calculated for $[\mathrm{M}+\mathrm{Na}]^{+}$ $\left(\mathrm{C}_{35} \mathrm{H}_{52} \mathrm{O}_{3} \mathrm{~N}_{2} \mathrm{Na}\right): \mathrm{m} / \mathrm{z} 571.39$, found: m/z 537.30.

\section{Fmoc-L-16}

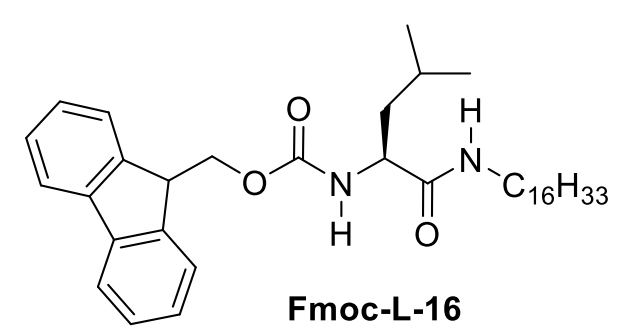

Yield: $536 \mathrm{mg}, 93 \% .{ }^{1} \mathrm{H}$ NMR (400 MHz, $\left.\mathrm{CDCl}_{3}\right) \delta 7.74(\mathrm{~d}, J=7.5 \mathrm{~Hz}, 2 \mathrm{H}), 7.55(\mathrm{~d}, J=$ $7.3 \mathrm{~Hz}, 2 \mathrm{H}), 7.38(\mathrm{t}, J=7.4 \mathrm{~Hz}, 2 \mathrm{H}), 7.29(\mathrm{t}, J=$ $7.4 \mathrm{~Hz}, 2 \mathrm{H}), 6.29(\mathrm{~s}, 1 \mathrm{H}), 5.51(\mathrm{~d}, J=8.1 \mathrm{~Hz}$, 1H), 4.46-4.35 (m, 2H), 4.23-4.13 (m, 2H), 3.31-3.10 (m, 2H), 1.71-1.60 (m, 2H), 1.58-1.51 (m, 1H), 1.50-1.41 (m, 2H), 1.33-1.18 (m, 26H), 0.99-0.84 (m, 9H). $\left.{ }^{13} \mathrm{C} \mathrm{NMR} \mathrm{(100} \mathrm{MHz,} \mathrm{CDCl}_{3}\right) \delta$ 172.14, 156.37, 143.81, 143.75, 141.32, 127.76, 127.11, 125.06, 120.01, 67.04, 53.63, 47.16, $41.58,39.62,31.97,29.74,29.70,29.64,29.59,29.50,29.41,29.31,26.91,24.74$, 22.92, 22.73, 22.19, 14.17. MS-ESI: calculated for $[\mathrm{M}+\mathrm{Na}]^{+}\left(\mathrm{C}_{37} \mathrm{H}_{56} \mathrm{O}_{3} \mathrm{~N}_{2} \mathrm{Na}\right): \mathrm{m} / \mathrm{z}$ 599.42, found: $\mathrm{m} / \mathrm{z} 599.31$. 


\section{Fmoc-I-12}

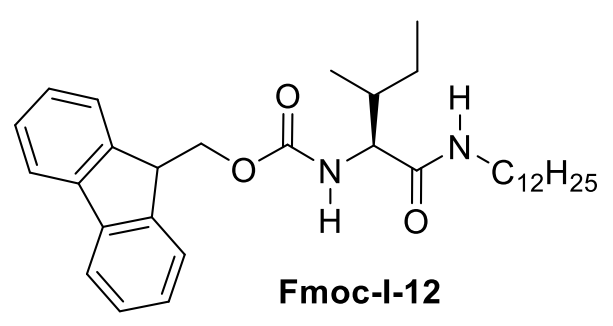

Yield: $499 \mathrm{mg}, 96 \% .{ }^{1} \mathrm{H}$ NMR (400 MHz, $\left.\mathrm{CDCl}_{3}\right) \delta 7.76(\mathrm{~d}, J=7.5 \mathrm{~Hz}, 2 \mathrm{H}), 7.58(\mathrm{~d}, J=$ $7.4 \mathrm{~Hz}, 2 \mathrm{H}), 7.40(\mathrm{t}, J=7.4 \mathrm{~Hz}, 2 \mathrm{H}), 7.31(\mathrm{t}, J=$ $7.4 \mathrm{~Hz}, 2 \mathrm{H}), 5.92$ (s, 1H), 5.46 (d, $J=8.0 \mathrm{~Hz}$, $1 \mathrm{H}), 4.47-4.32(\mathrm{~m}, 2 \mathrm{H}), 4.21(\mathrm{t}, J=6.9 \mathrm{~Hz}, 1 \mathrm{H})$, $3.93(\mathrm{t}, J=7.8 \mathrm{~Hz}, 1 \mathrm{H}), 3.34-3.11(\mathrm{~m}, 2 \mathrm{H}), 1.94-1.82(\mathrm{~m}, 1 \mathrm{H}), 1.57-1.42(\mathrm{~m}, 3 \mathrm{H})$, 1.32-1.21 (m, 18H), 1.19-1.07 (m, 1H), 0.97-0.84 (m, 9H). ${ }^{13} \mathrm{C}$ NMR (100 MHz, $\left.\mathrm{CDCl}_{3}\right) \delta 171.06,156.32,143.77,143.73,141.27,127.70,127.06,125.06,125.03$, $119.97,66.97,59.85,47.13,39.57,37.25,31.88,29.60,29.54,29.50,29.45,29.32$, $29.20,26.85,24.82,22.66,15.45,14.10,11.28$. MS-ESI: calculated for $[\mathrm{M}+\mathrm{Na}]^{+}$ $\left(\mathrm{C}_{33} \mathrm{H}_{48} \mathrm{O}_{3} \mathrm{~N}_{2} \mathrm{Na}\right): \mathrm{m} / \mathrm{z}$ 543.36, found: m/z 543.30.

\section{Fmoc-I-14}

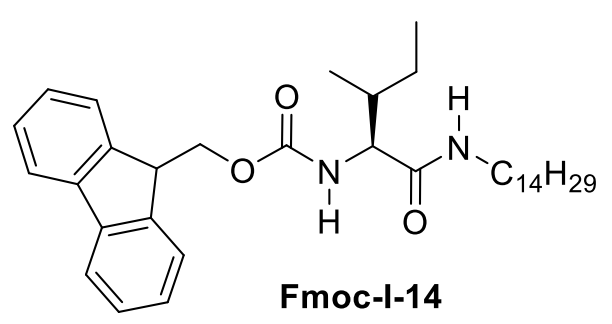

Yield: $532 \mathrm{mg}$, 97\%. ${ }^{1} \mathrm{H}$ NMR (400 MHz, $\left.\mathrm{CDCl}_{3}\right) \delta 7.76(\mathrm{~d}, J=7.5 \mathrm{~Hz}, 2 \mathrm{H}), 7.58(\mathrm{~d}, J=$ $7.4 \mathrm{~Hz}, 2 \mathrm{H}), 7.40$ (t, $J=7.4 \mathrm{~Hz}, 2 \mathrm{H}), 7.31(\mathrm{t}, J=$ $7.4 \mathrm{~Hz}, 2 \mathrm{H}), 5.91(\mathrm{~s}, 1 \mathrm{H}), 5.45(\mathrm{~d}, J=8.0 \mathrm{~Hz}$, $1 \mathrm{H}), 4.46-4.32(\mathrm{~m}, 2 \mathrm{H}), 4.21(\mathrm{t}, J=6.9 \mathrm{~Hz}, 1 \mathrm{H})$, $3.93(\mathrm{t}, J=7.8 \mathrm{~Hz}, 1 \mathrm{H}), 3.36-3.13(\mathrm{~m}, 2 \mathrm{H}), 1.94-1.82(\mathrm{~m}, 1 \mathrm{H}), 1.58-1.41(\mathrm{~m}, 3 \mathrm{H})$, 1.33-1.20 (m, 22H), 1.18-1.05 (m, 1H), 0.97-0.84 (m, 9H). ${ }^{13} \mathrm{C}$ NMR (100 MHz, $\left.\mathrm{CDCl}_{3}\right) \delta 171.11,156.39,143.83,143.79,141.33,127.76,127.12,125.12,125.09$, $120.03,67.03,59.91,47.19,39.62,37.33,31.95,29.68,29.61,29.57,29.52,29.40$, 29.27, 26.91, 24.88, 22.73, 15.51, 14.17, 11.35. MS-ESI: calculated for $[\mathrm{M}+\mathrm{Na}]^{+}$ $\left(\mathrm{C}_{35} \mathrm{H}_{52} \mathrm{O}_{3} \mathrm{~N}_{2} \mathrm{Na}\right): \mathrm{m} / \mathrm{z}$ 571.39, found: m/z 571.29. 


\section{Fmoc-I-16}

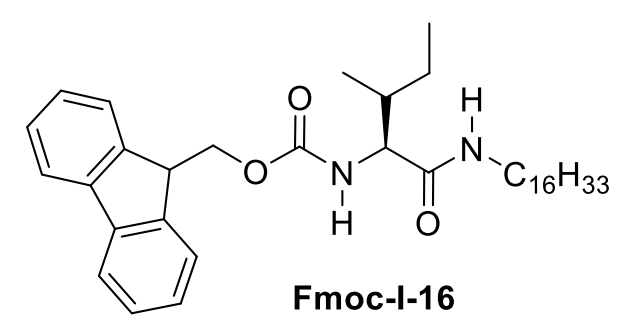

Yield: $559 \mathrm{mg}, 97 \% .{ }^{1} \mathrm{H}$ NMR $(400 \mathrm{MHz}$, $\left.\mathrm{CDCl}_{3}\right) \delta 7.76(\mathrm{~d}, J=7.5 \mathrm{~Hz}, 2 \mathrm{H}), 7.58(\mathrm{~d}, J=$ $7.4 \mathrm{~Hz}, 2 \mathrm{H}), 7.40(\mathrm{t}, J=7.4 \mathrm{~Hz}, 2 \mathrm{H}), 7.31(\mathrm{t}, J=$ $7.4 \mathrm{~Hz}, 2 \mathrm{H}), 5.82(\mathrm{~s}, 1 \mathrm{H}), 5.40(\mathrm{~d}, J=8.5 \mathrm{~Hz}$, $1 \mathrm{H}), 4.49-4.33(\mathrm{~m}, 2 \mathrm{H}), 4.21(\mathrm{t}, J=6.9 \mathrm{~Hz}, 1 \mathrm{H})$, $3.93(\mathrm{t}, J=7.8 \mathrm{~Hz}, 1 \mathrm{H}), 3.37-3.13(\mathrm{~m}, 2 \mathrm{H}), 1.93-1.80(\mathrm{~m}, 1 \mathrm{H}), 1.56-1.43(\mathrm{~m}, 3 \mathrm{H})$, 1.33-1.21 (m, 26H), 1.18-1.08 (m, 1H), 0.98-0.84 (m, 9H). ${ }^{13} \mathrm{C}$ NMR (100 MHz, $\left.\mathrm{CDCl}_{3}\right) \delta 143.84,141.34,127.76,127.12,125.10,120.02,67.00,59.93,47.22,39.59$, $37.34,31.95,29.72$, 29.70, 29.69, 29.60, 29.56, 29.53, 29.39, 29.26, 26.91, 24.87, 22.72, 15.51, 14.15, 11.35. MS-ESI: calculated for $[\mathrm{M}+\mathrm{Na}]^{+}\left(\mathrm{C}_{37} \mathrm{H}_{56} \mathrm{O}_{3} \mathrm{~N}_{2} \mathrm{Na}\right): \mathrm{m} / \mathrm{z}$ 599.42, found: $\mathrm{m} / \mathrm{z} 599.32$.

\section{Fmoc-F-12}

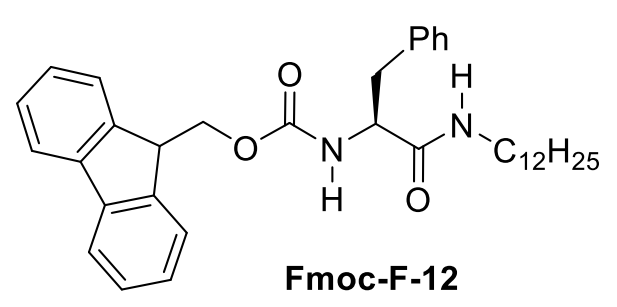

Yield: $526 \mathrm{mg}$, 95\%. ${ }^{1} \mathrm{H}$ NMR (400 MHz, $\left.\mathrm{CDCl}_{3}\right) \delta 7.76(\mathrm{~d}, J=7.5 \mathrm{~Hz}, 2 \mathrm{H}), 7.55(\mathrm{dd}, J=$ 7.2, $6.4 \mathrm{~Hz}, 2 \mathrm{H}), 7.40(\mathrm{t}, J=7.4 \mathrm{~Hz}, 2 \mathrm{H})$, 7.34-7.16 (m, 7H), $5.58(\mathrm{~s}, 1 \mathrm{H}), 5.47$ (d, $J=6.4$ $\mathrm{Hz}, 1 \mathrm{H}), 4.47-4.38(\mathrm{~m}, 1 \mathrm{H}), 4.37-4.28(\mathrm{~m}, 2 \mathrm{H})$, $4.19(\mathrm{t}, J=6.8 \mathrm{~Hz}, 1 \mathrm{H}), 3.20-2.93(\mathrm{~m}, 4 \mathrm{H}), 1.33-1.18(\mathrm{~m}, 18 \mathrm{H}), 1.17-1.09(\mathrm{~m}, 2 \mathrm{H})$, $0.88(\mathrm{t}, J=6.7 \mathrm{~Hz}, 3 \mathrm{H}) .{ }^{13} \mathrm{C} \mathrm{NMR}\left(100 \mathrm{MHz}, \mathrm{CDCl}_{3}\right) \delta 170.60,155.99,143.77$, 141.33, 136.69, 129.36, 128.71, 127.78, 127.12, 127.06, 125.06, 120.03, 67.08, 56.54, $47.13,39.59,39.03,31.96,29.69,29.63,29.55,29.39,29.33,29.28,26.82,22.74$, 14.18. MS-ESI: calculated for $[\mathrm{M}+\mathrm{Na}]^{+}\left(\mathrm{C}_{36} \mathrm{H}_{46} \mathrm{O}_{3} \mathrm{~N}_{2} \mathrm{Na}\right): \mathrm{m} / \mathrm{z} 577.34$, found: $\mathrm{m} / \mathrm{z}$ 577.24 . 


\section{Fmoc-F-14}

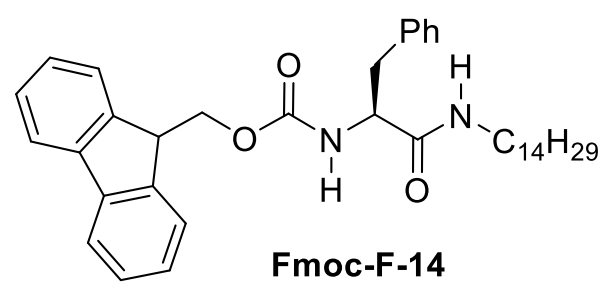

Yield: $553 \mathrm{mg}$, 95\%. ${ }^{1} \mathrm{H}$ NMR (400 MHz, $\left.\mathrm{CDCl}_{3}\right) \delta 7.76(\mathrm{~d}, J=7.5 \mathrm{~Hz}, 2 \mathrm{H}), 7.55(\mathrm{dd}, J=$ $7.2,4.9 \mathrm{~Hz}, 2 \mathrm{H}), 7.40(\mathrm{t}, J=7.4 \mathrm{~Hz}, 2 \mathrm{H})$, 7.34-7.15 (m, 7H), $5.60(\mathrm{~s}, 1 \mathrm{H}), 5.49(\mathrm{~d}, J=8.2$ $\mathrm{Hz}, 1 \mathrm{H}), 4.46-4.37(\mathrm{~m}, 1 \mathrm{H}), 4.36-4.28(\mathrm{~m}, 2 \mathrm{H})$, $4.19(\mathrm{t}, J=6.9 \mathrm{~Hz}, 1 \mathrm{H}), 3.20-3.05(\mathrm{~m}, 3 \mathrm{H}), 3.04-2.94(\mathrm{~m}, 1 \mathrm{H}), 1.35-1.18(\mathrm{~m}, 22 \mathrm{H})$, 1.17-1.08 (m, 2H), $0.88(\mathrm{t}, J=6.7 \mathrm{~Hz}, 3 \mathrm{H}) .{ }^{13} \mathrm{C} \mathrm{NMR}\left(100 \mathrm{MHz}, \mathrm{CDCl}_{3}\right) \delta 170.55$, $155.98,143.77,141.33,136.67,129.35,128.73$, 127.78, 127.12, 127.07, 125.05, 120.03, 67.06, 56.54, 47.14, 39.59, 39.01, 31.96, 29.74, 29.72, 29.69, 29.63, 29.54, $29.40,29.32,29.27,26.81,22.73$, 14.17. MS-ESI: calculated for $[\mathrm{M}+\mathrm{Na}]^{+}$ $\left(\mathrm{C}_{38} \mathrm{H}_{50} \mathrm{O}_{3} \mathrm{~N}_{2} \mathrm{Na}\right): \mathrm{m} / \mathrm{z} 605.37$, found: m/z 605.26.

\section{Fmoc-F-16}

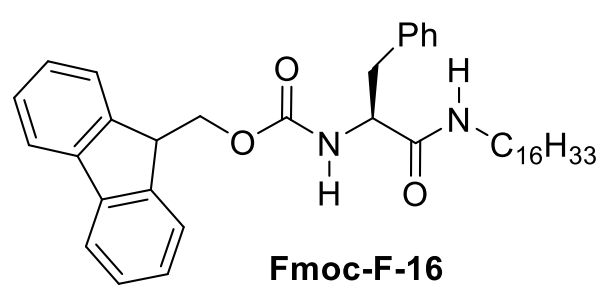

Yield: $586 \mathrm{mg}, 96 \% .{ }^{1} \mathrm{H}$ NMR (400 MHz, $\left.\mathrm{CDCl}_{3}\right) \delta 7.76(\mathrm{~d}, J=7.5 \mathrm{~Hz}, 2 \mathrm{H}), 7.54(\mathrm{dd}, J=$ 7.0, $5.2 \mathrm{~Hz}, 2 \mathrm{H}), 7.40(\mathrm{t}, J=7.4 \mathrm{~Hz}, 2 \mathrm{H})$, 7.34-7.17 (m, 7H), 5.57 (s, 1H), 5.47 (d, $J=6.1$ $\mathrm{Hz}, 1 \mathrm{H}), 4.47-4.38(\mathrm{~m}, 1 \mathrm{H}), 4.37-4.28(\mathrm{~m}, 2 \mathrm{H})$, $4.19(\mathrm{t}, J=6.8 \mathrm{~Hz}, 1 \mathrm{H}), 3.21-3.04(\mathrm{~m}, 3 \mathrm{H}), 3.03-2.93(\mathrm{~m}, 1 \mathrm{H}), 1.34-1.17(\mathrm{~m}, 26 \mathrm{H})$, 1.16-1.08 (m, 2H), $0.88(\mathrm{t}, J=6.7 \mathrm{~Hz}, 3 \mathrm{H}) .{ }^{13} \mathrm{C} \mathrm{NMR}\left(100 \mathrm{MHz}, \mathrm{CDCl}_{3}\right) \delta 170.45$, $155.95,143.75,141.34,136.64,129.34,128.76,127.78,127.12,125.04,120.04$, 67.04, 56.54, 47.16, 39.59, 38.99, 31.96, 29.73, 29.72, 29.69, 29.62, 29.53, 29.39, 29.31, 29.26, 26.80, 22.72, 14.16. MS-ESI: calculated for $[\mathrm{M}+\mathrm{Na}]^{+}\left(\mathrm{C}_{40} \mathrm{H}_{54} \mathrm{O}_{3} \mathrm{~N}_{2} \mathrm{Na}\right)$ : $\mathrm{m} / \mathrm{z}$ 633.40, found: $\mathrm{m} / \mathrm{z} 633.29$. 

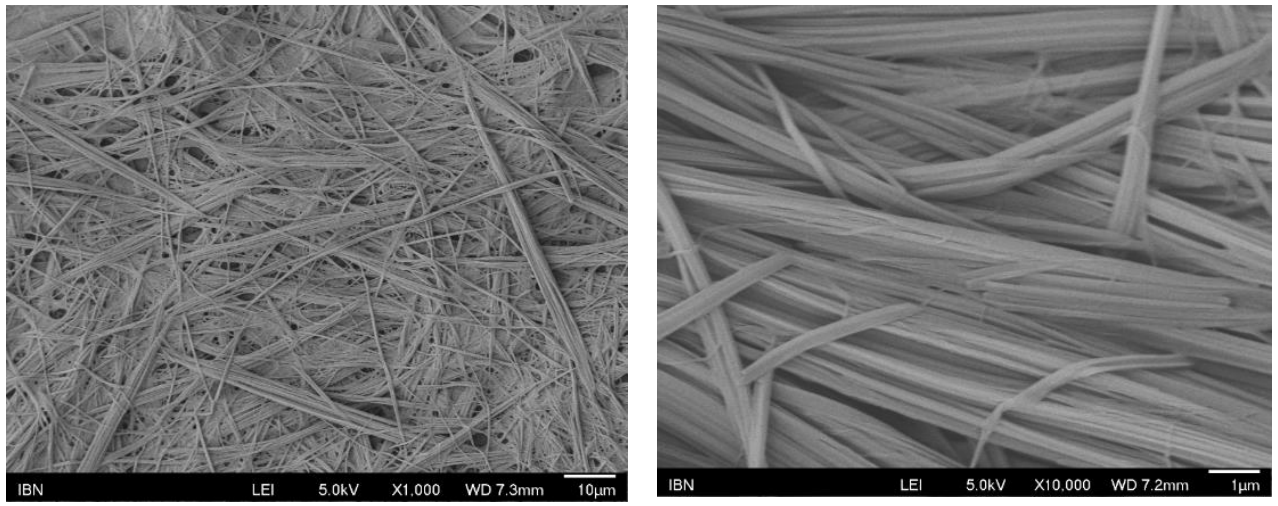

Toluene Gel
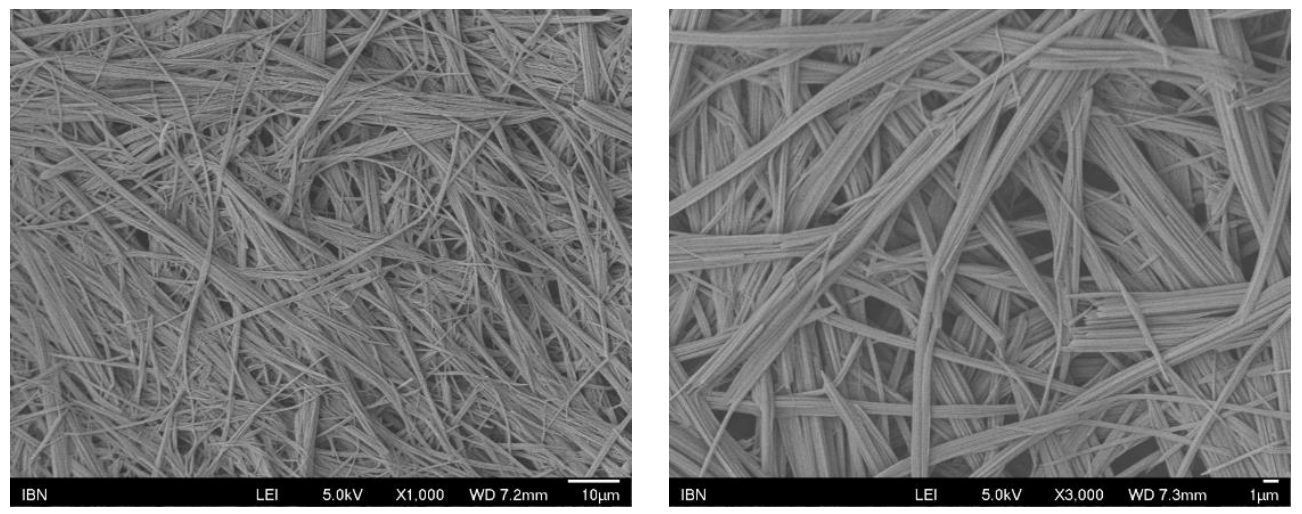

Xylene Gel

Figure S1. SEM images of the as-formed toluene and xylene gels by Fmoc-V-6. 


\section{${ }^{1} \mathrm{H}$ NMR and ${ }^{13} \mathrm{C}$ NMR}

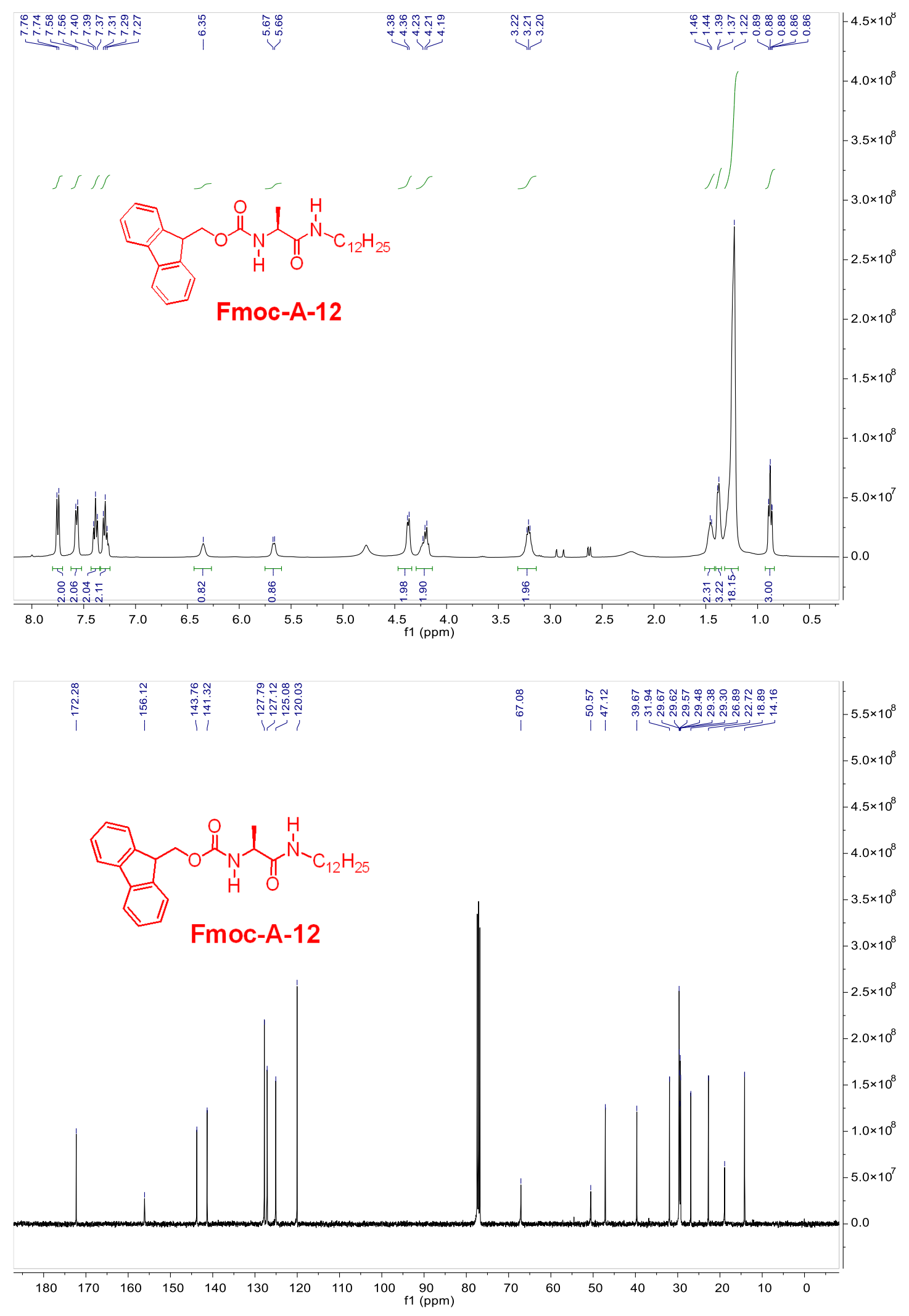




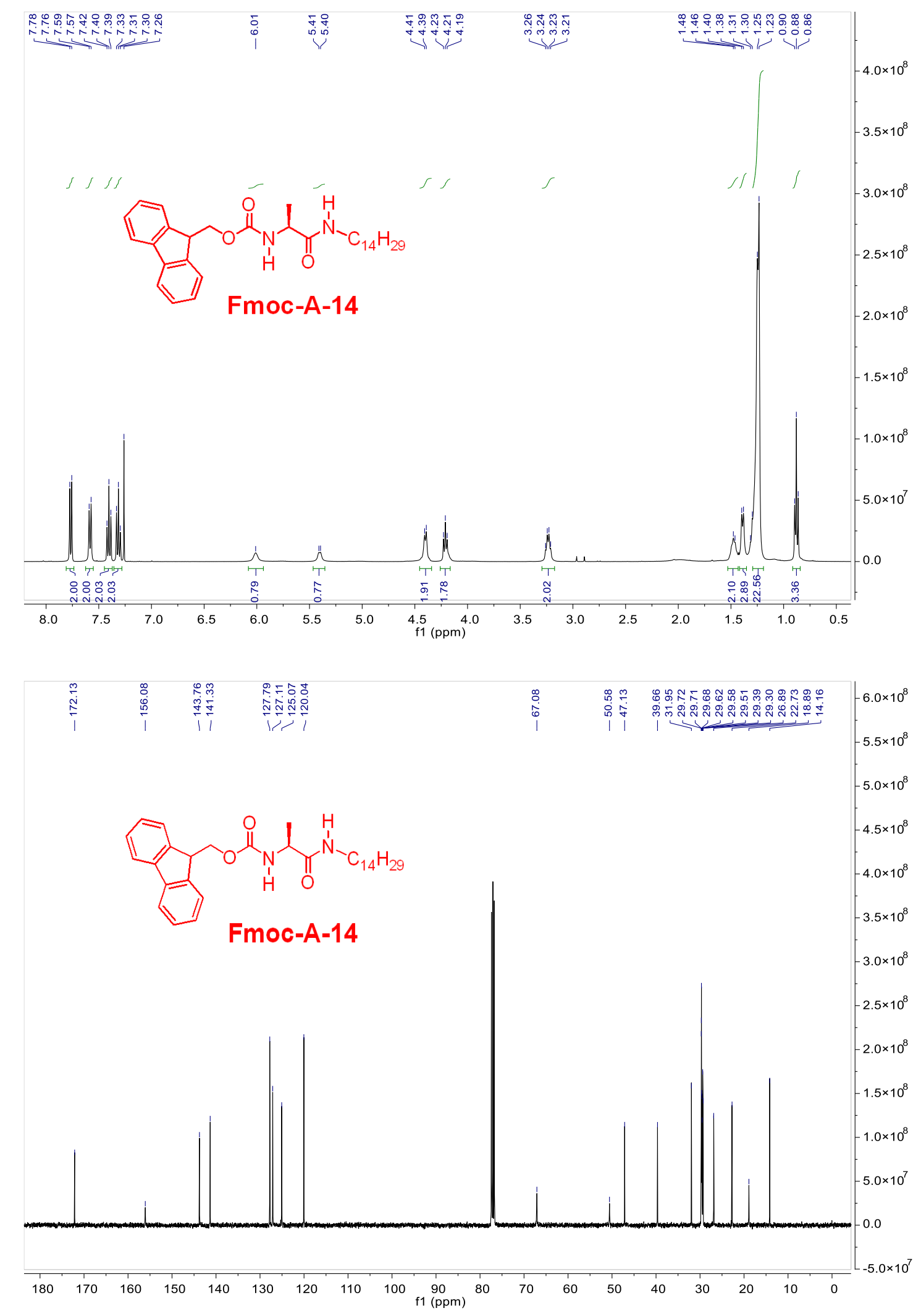




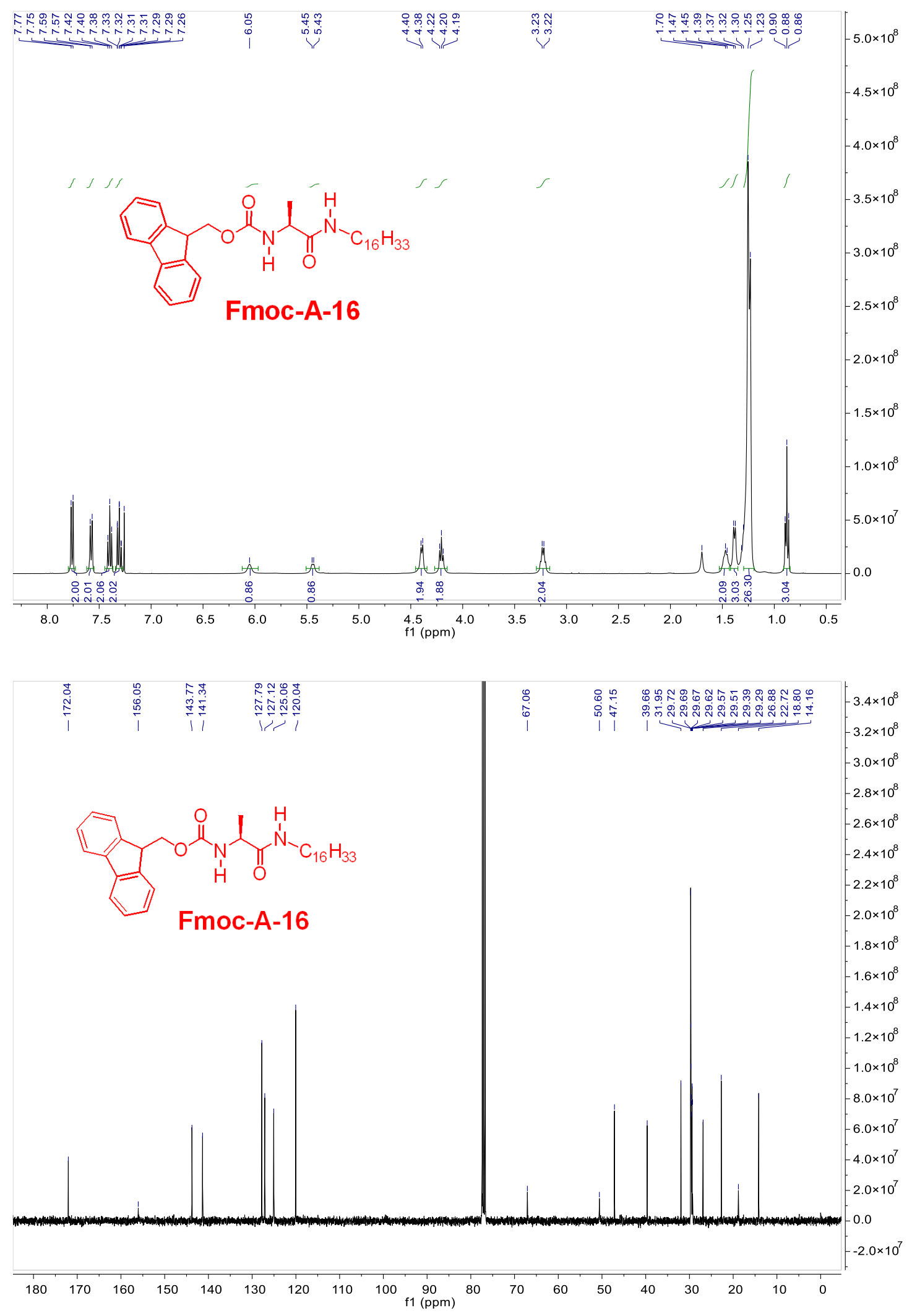




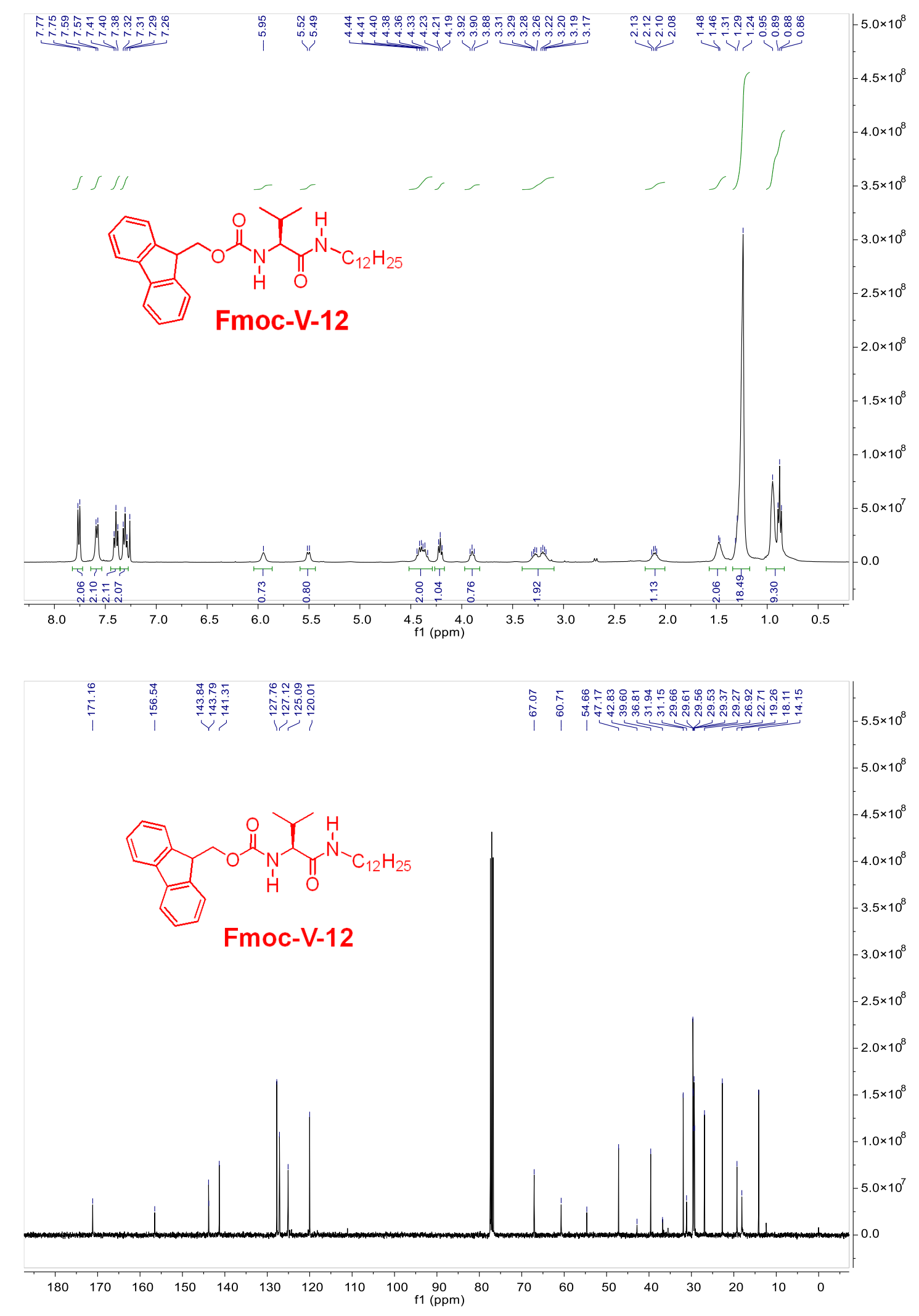




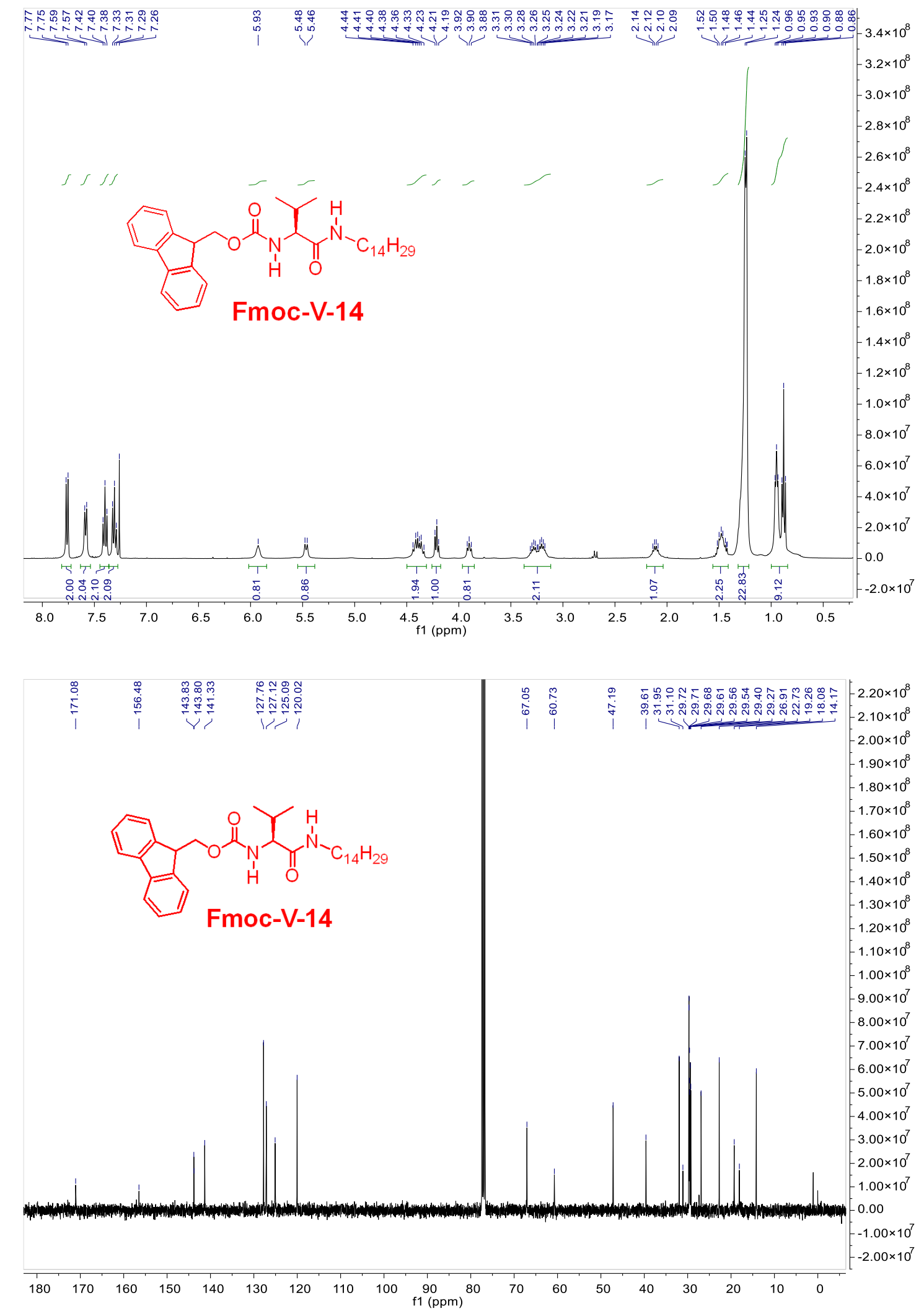




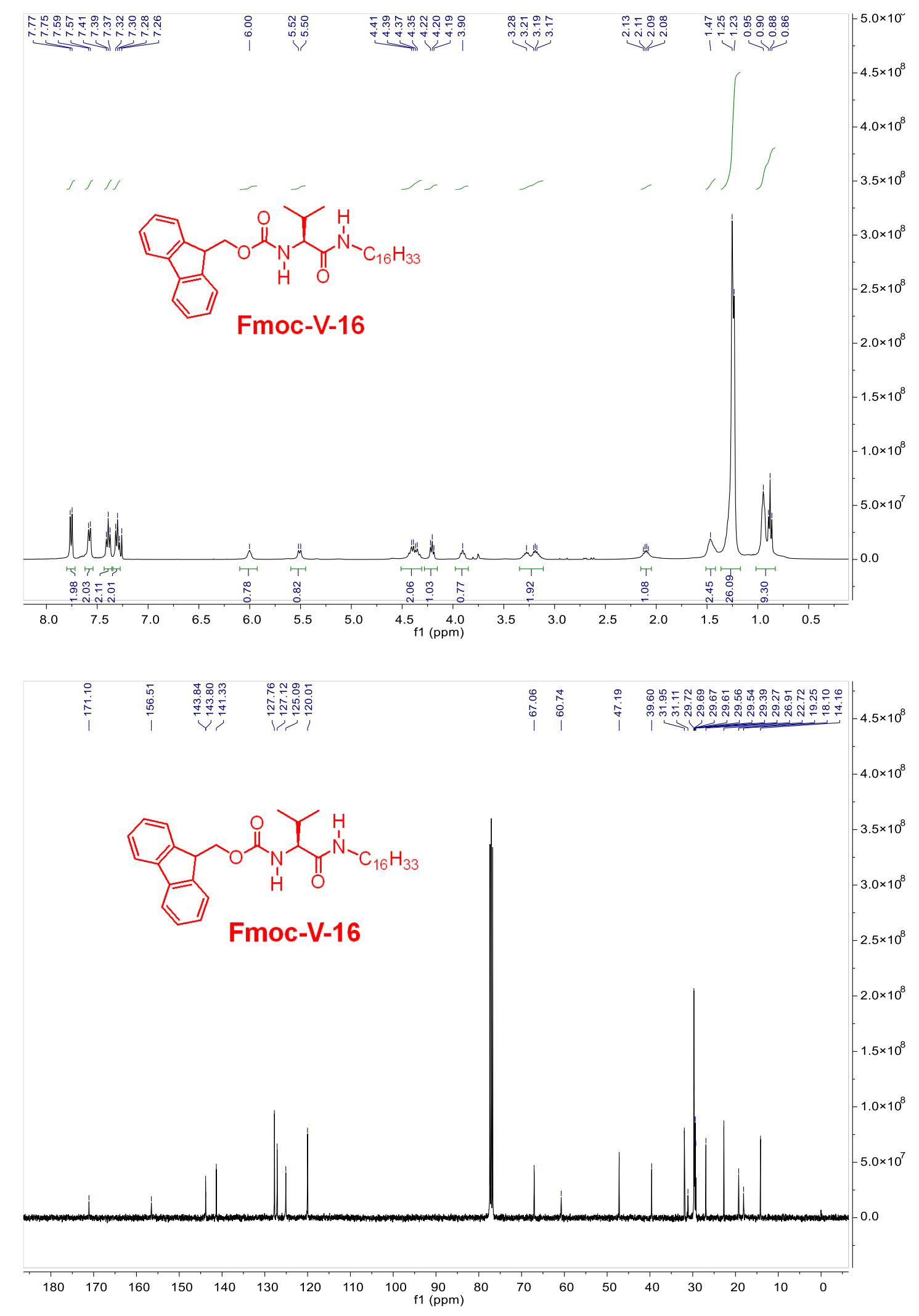




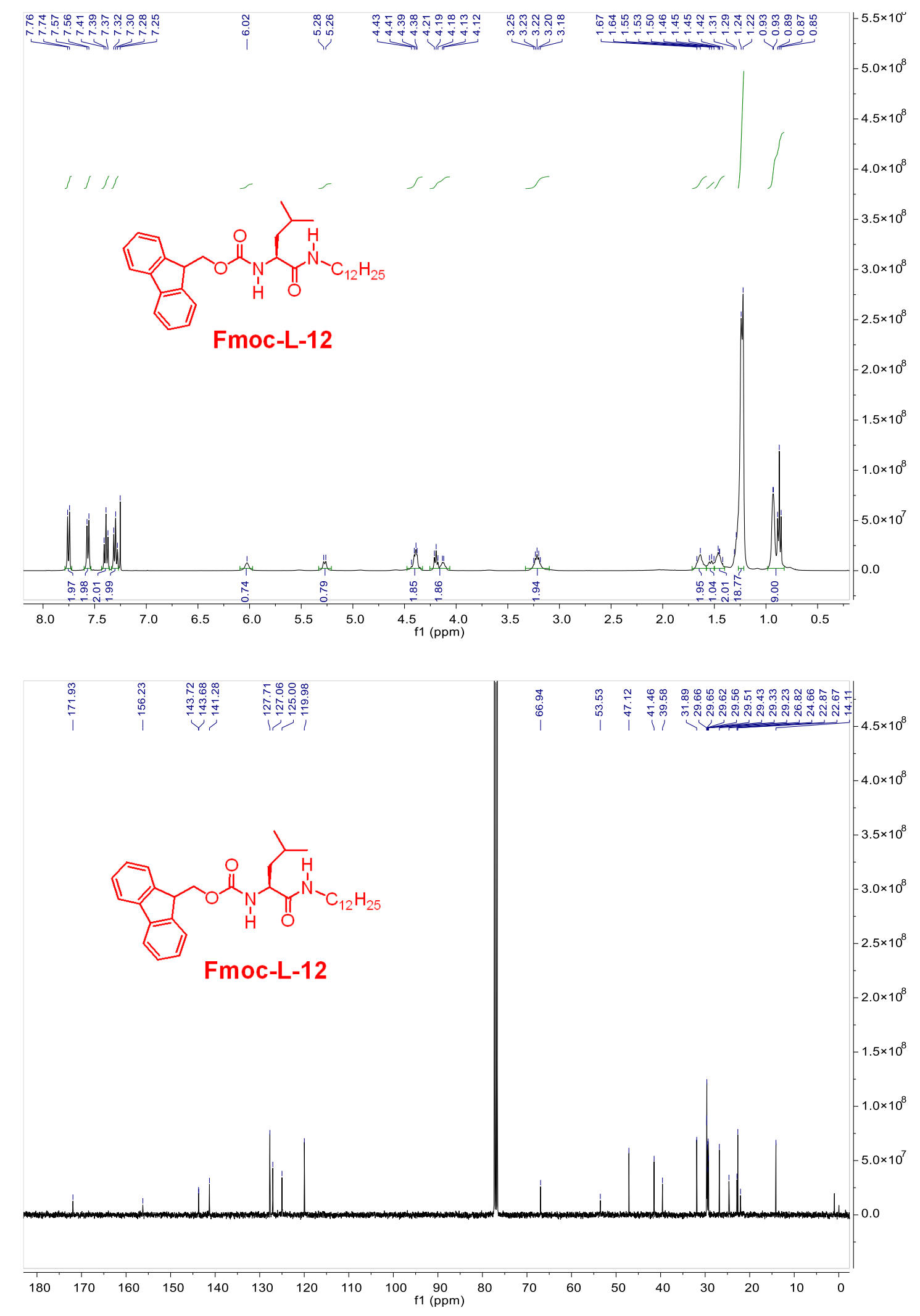




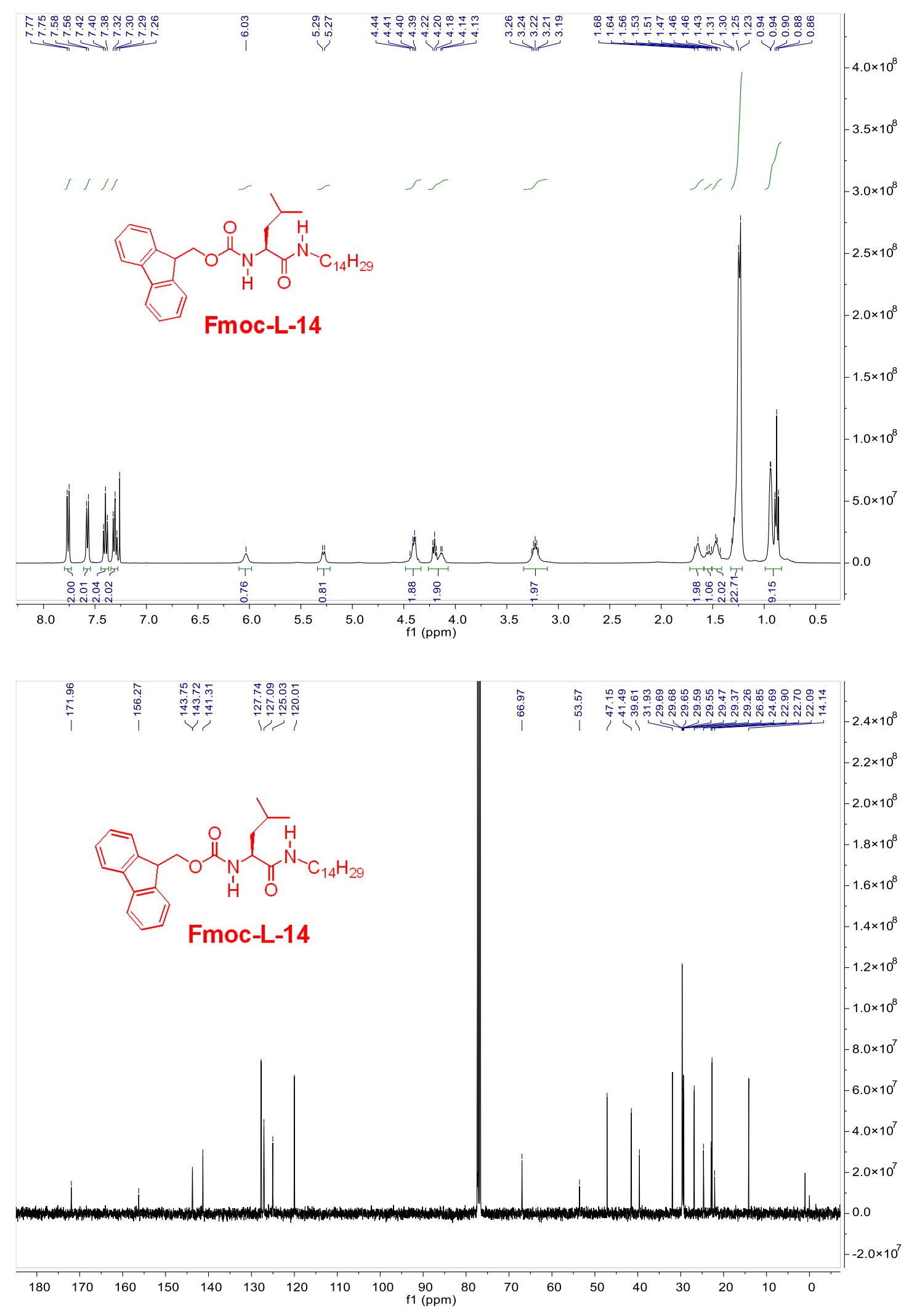




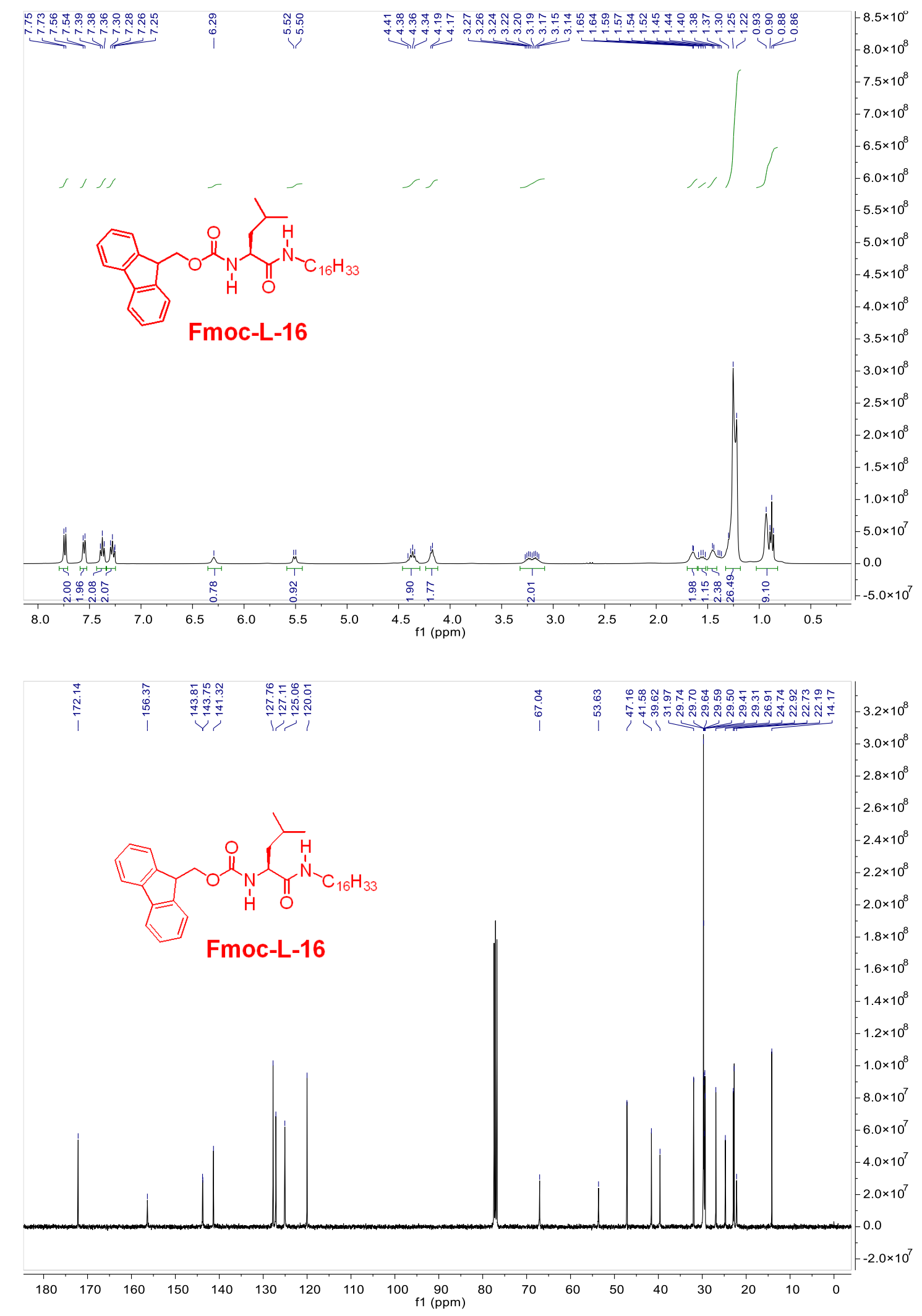




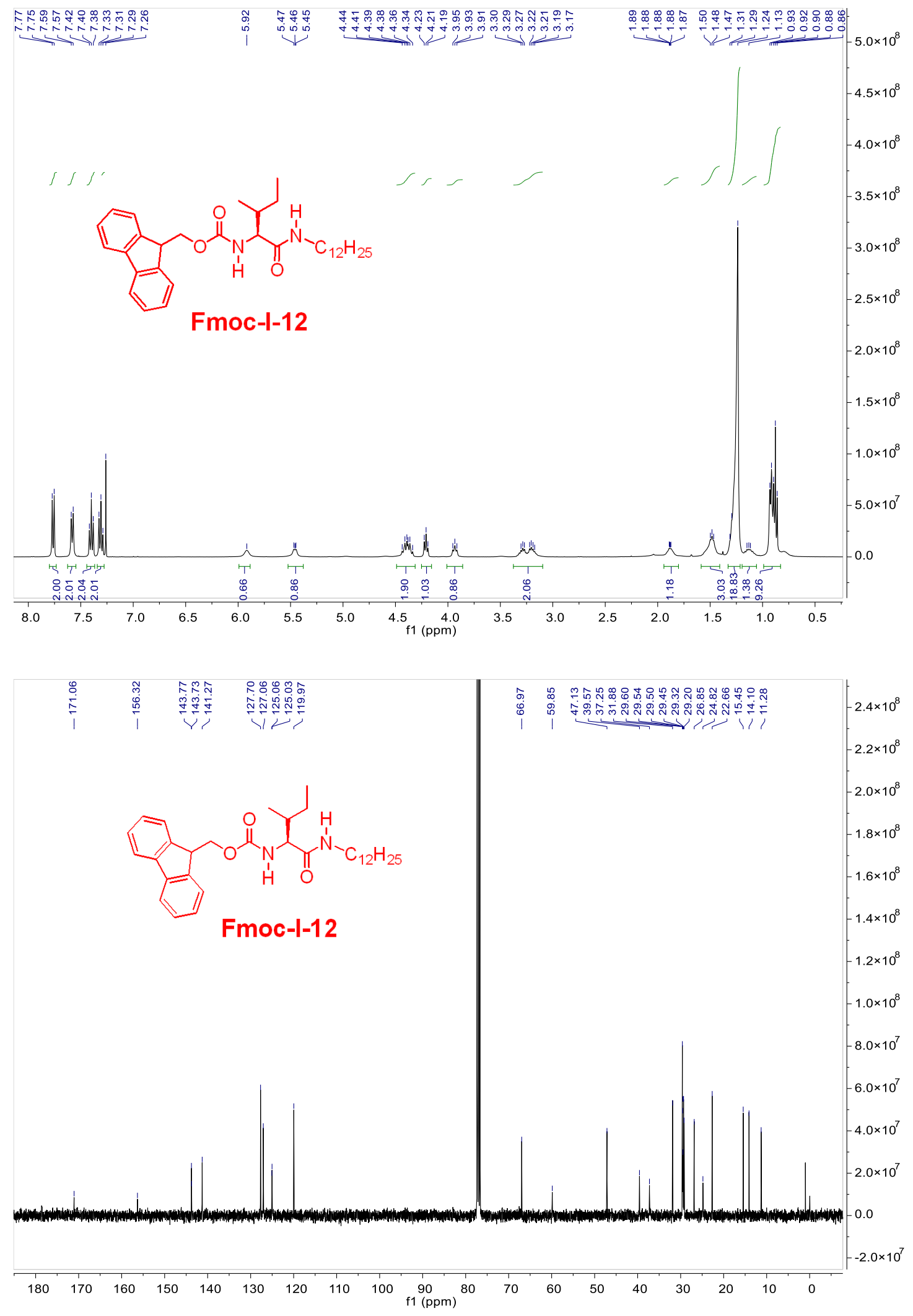




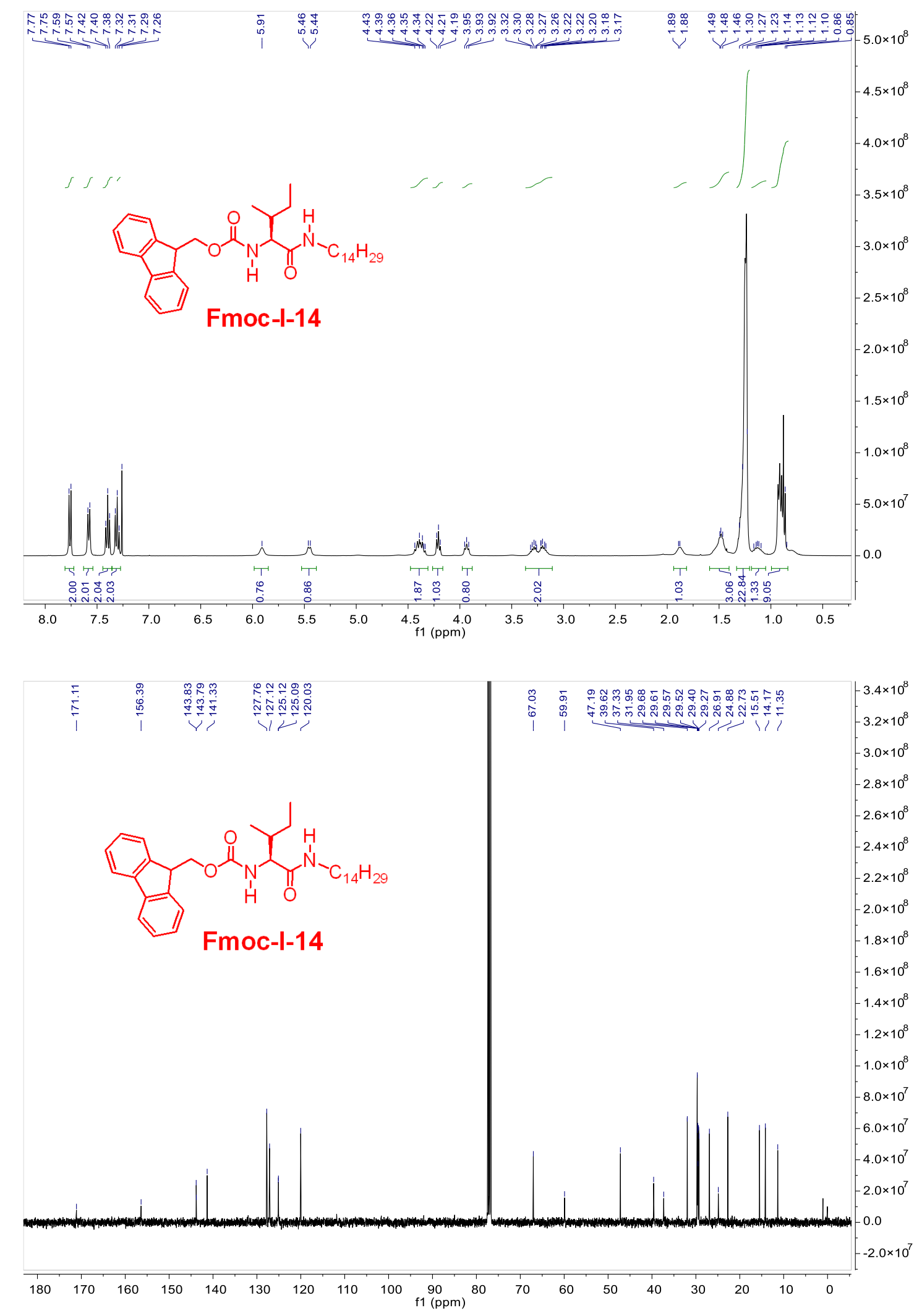




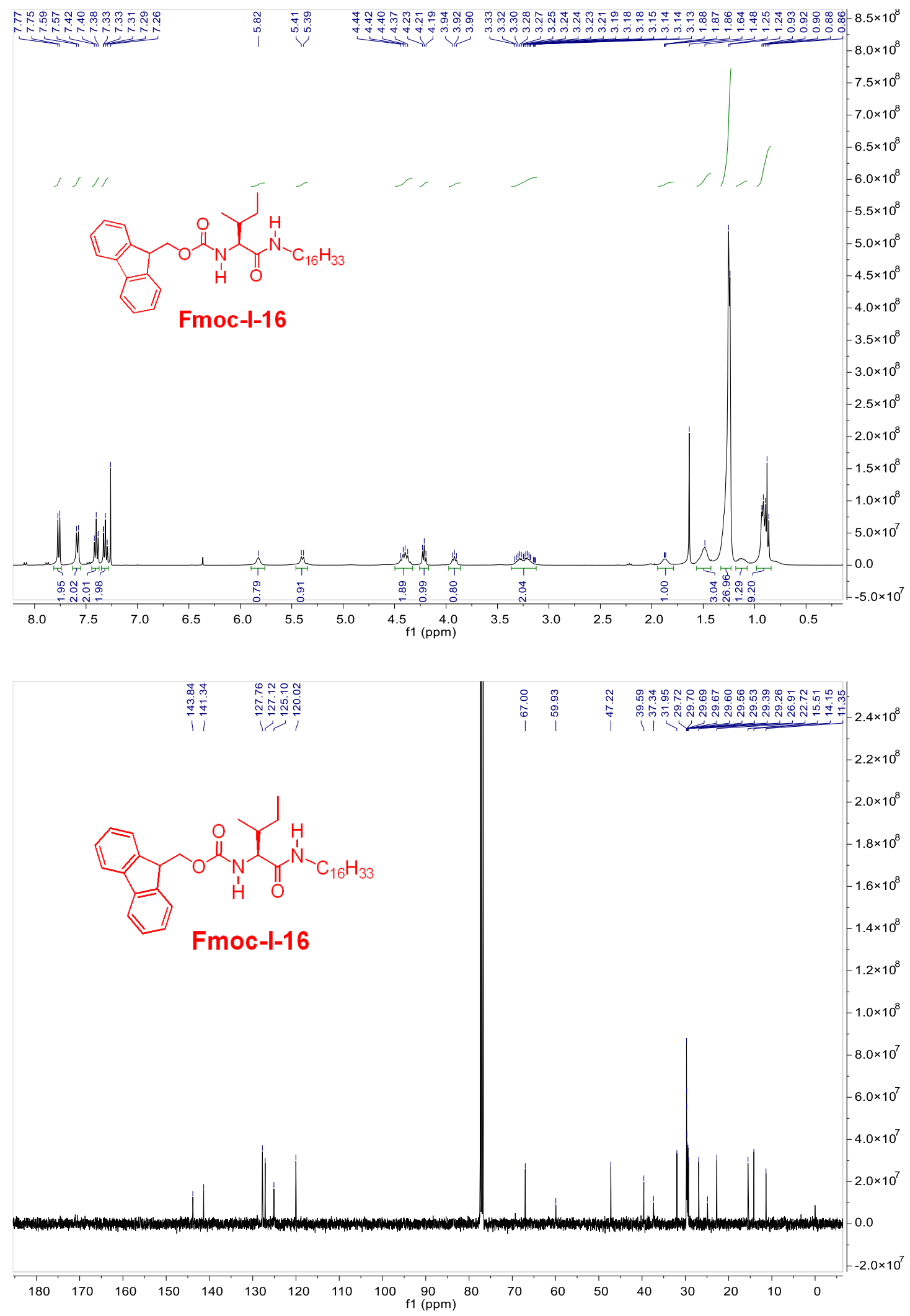




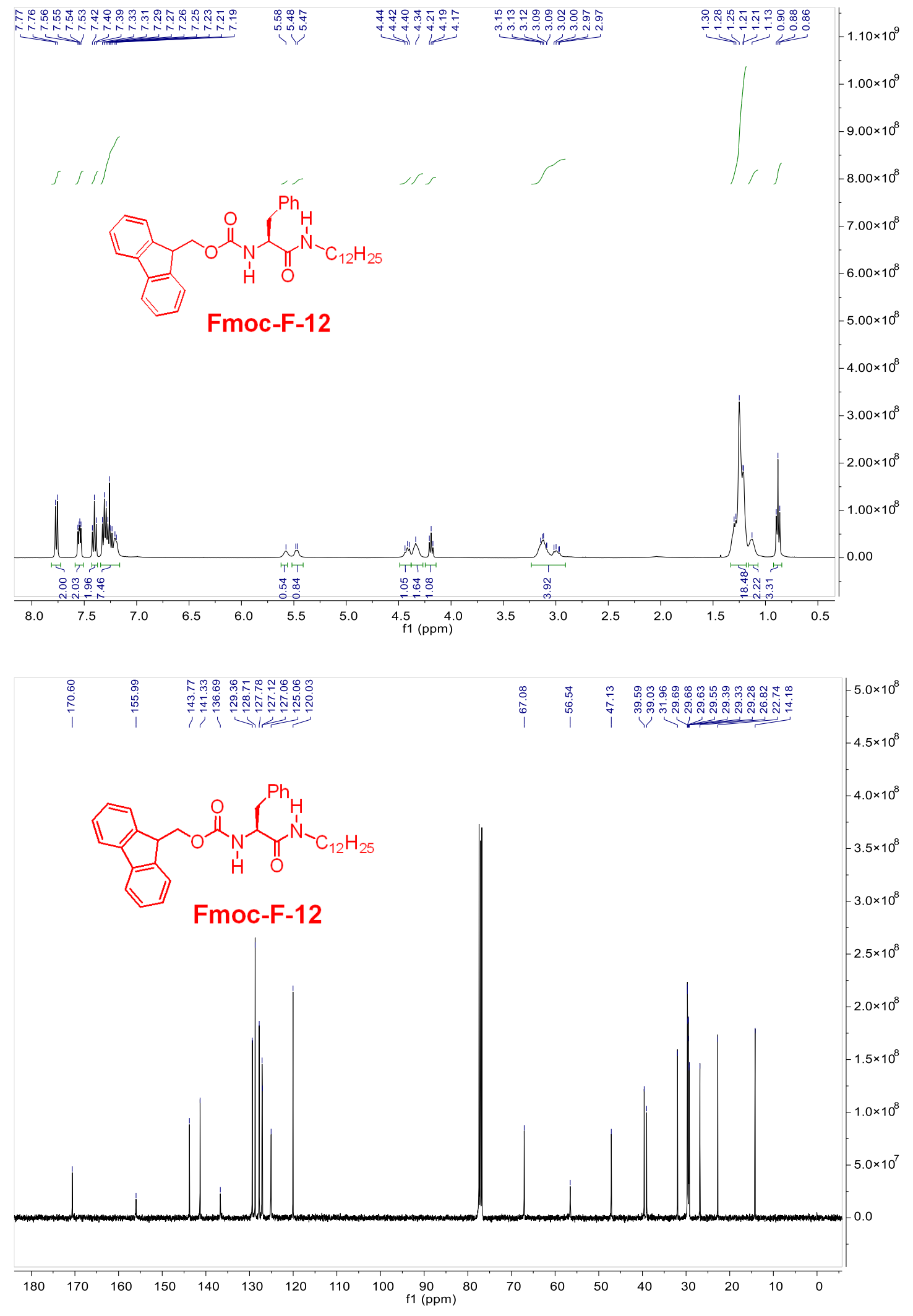




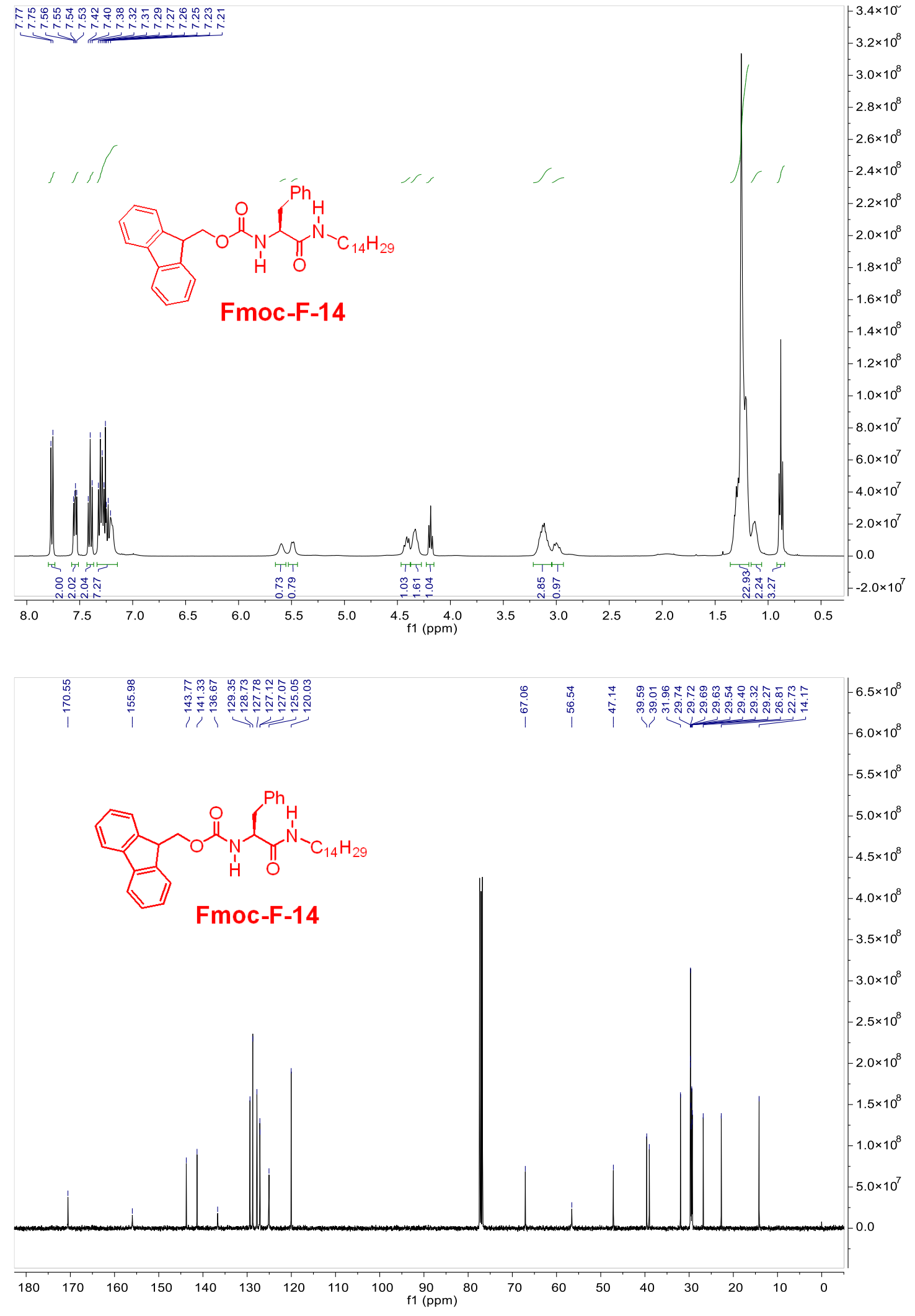




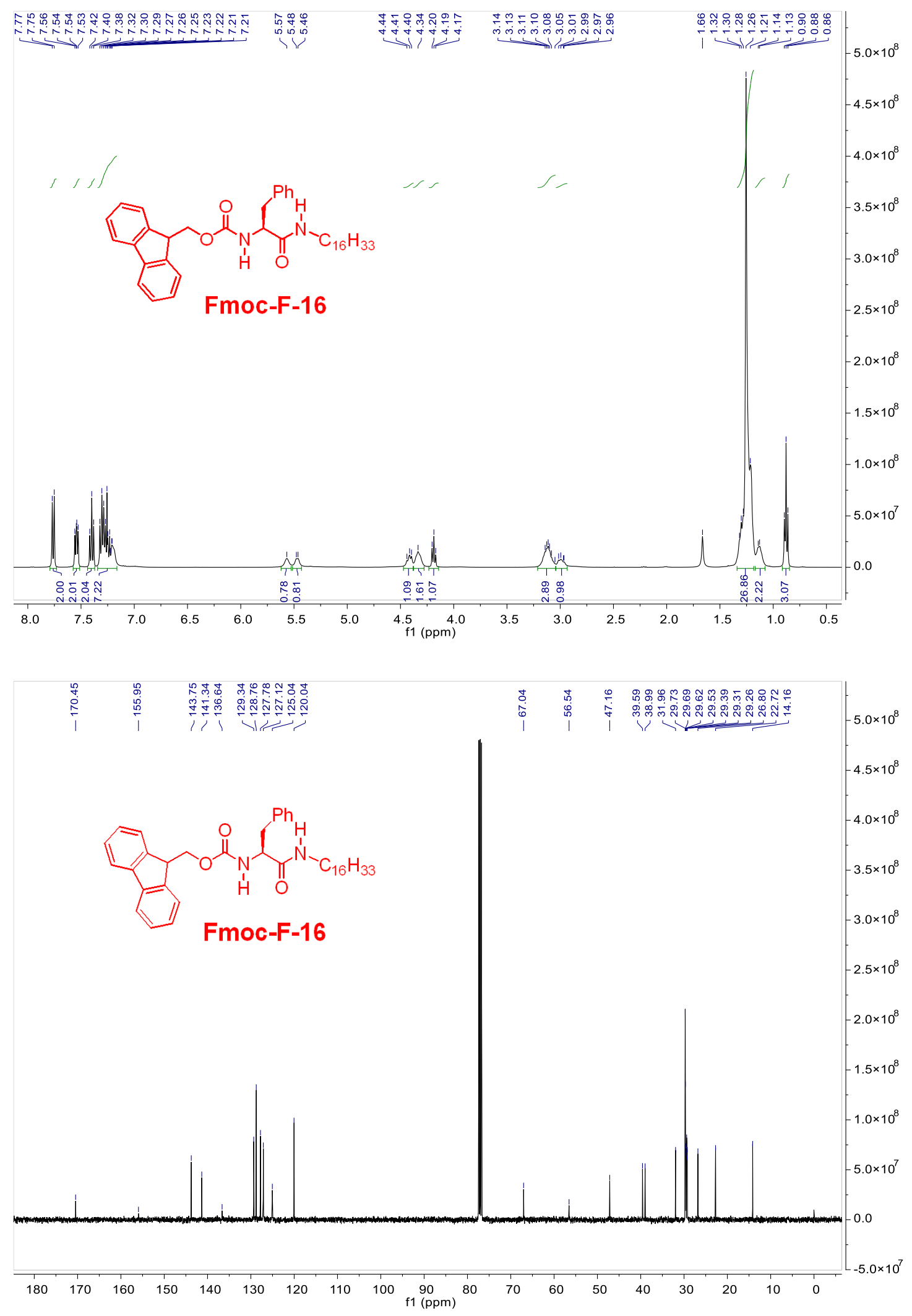

